INR-TH-2021-025

\title{
Constraints on the models of the origin of high-energy astrophysical neutrinos ${ }^{*}$
}

\author{
Sergey Troitsky \\ Institute for Nuclear Research of the Russian Academy of Sciences, \\ 60th October Anniversary Prospect 7a, Moscow 117312, Russia
}

(Dated: Received July 2, revised September 7, accepted September 13, 2021)

\begin{abstract}
The existence of astrophysical neutrinos with energies of tens of $\mathrm{TeV}$ and higher has been reliably established by the IceCube experiment; the first confirmations of this discovery are being obtained with the ANTARES and Baikal-GVD facilities. The observational results do not fully agree with what was expected before the start of these experiments. The origin of these neutrinos has not been conclusively established, and simple theoretical models, popular for decades, cannot explain all observational data. This review summarizes the experimental results with emphasis on those important for constraining theoretical models, discusses various scenarios for the origin of high-energy neutrinos and briefly lists particualr classes of their potential astrophysical sources. It is demonstrated that the observational data may be explained if the flux of astrophysical neutrinos includes the contribution of extragalactic sources, dominating at the highest energies, and the Galactic component, significant only at neutrino energies $\lesssim 100 \mathrm{TeV}$. Other possible scenarios are also discussed.
\end{abstract}

\section{CONTENTS}

1. Introduction

1.1. Astrophysical high-energy neutrinos: formulation of the problem

1.2. High-energy neutrino detection

1.3. Past, present, and future experiments

2. Key experimental results

2.1. Extraterrestrial origin of neutrinos

2.2. Spectrum and flavor composition

2.3. Arrival directions

2.3.1. Constraints on the Galactic anisotropy 10

2.3.2. "Blind" search for point sources

2.3.3. Source populations

2.3.4. Search for flares

3. General constraints on models of neutrino origin 18

3.1. The $\pi$-meson mechanism and the multimessenger approach

3.2. General constraints on source populations

3.3. Conclusions about general constraints

4. Potential source classes

4.1. Models of extragalactic sources

4.2. Models of the Galactic flux component

5. Conclusions

References

* Invited review published in Physics Uspekhi in a special issue dedicated to the 50th anniversary of INR RAS, Russian doi:10.3367/UFNr.2021.09.039062, English doi:10.3367/UFNe.2021.09.039062.

$\dagger$ st@ms2.inr.ac.ru

\section{INTRODUCTION}

\subsection{Astrophysical high-energy neutrinos: formulation of the problem}

Modern astrophysics has confidently moved beyond the so-called photon channel, the study of sources based on the electromagnetic radiation of various bands coming from them. The first extraterrestrial sources of neutrinos, the Sun and supernova 1987A, and then also gravitational waves have been detected. Extraterrestrial charged particles, cosmic rays, are being studied intensively. Along with the development of electromagnetic astronomy, including the highest energies at which telescopes detect individual photons, this gave rise to the socalled multimessenger astrophysics, which uses different carriers - photons, neutrinos, charged particles and gravitational waves - to obtain information about the structure of astrophysical objects and about physical processes going on there. Here we will focus on one of the channels of the multimessenger astronomy, detection of highenergy neutrinos, and on related observations in other channels.

Due to the unique place of the neutrino among elementary particles (a stable particle experiencing weak interactions only), the main goal of the neutrino astronomy in the 20th century was the study of sources opaque to electromagnetic radiation. Thus, the discovery of solar neutrinos experimentally proved that the energy of the Sun comes from thermonuclear reactions in its central region not accessible to other observations, and the registration of neutrinos from the supernova 1987A allowed one to verify understanding of physical processes taking place in the interior of a massive star at the gravitational collapse of its core. In both cases neutrinos are born in nuclear processes and have energies corresponding to the characteristic nuclear scales (from fractions to tens of 
$\mathrm{MeV}$ ). Occurring in substantially opaque regions, these processes "heat" the source and are eventually related to the thermal electromagnetic radiation - for example, the solar radiation. At the same time a large fraction of the photon radiation in the Universe is associated with non-thermal processes and is determined by the interaction of relativistic particles with ambient fields, matter and radiation. As a rule, such nonthermal emission at relatively low energies, from radio to ultraviolet, and sometimes up to the X-ray band, is well explained by the synchrotron radiation of relativistic electrons. At higher energies, the situation becomes less clear - along with the synchrotron radiation of electrons, the inverse-Compton radiation may be significant, as well as the proton synchrotron or photon production in elementary-particle interactions. Since the only way to produce astrophysical neutrinos with energies $\gtrsim 10 \mathrm{GeV}$ that does not involve non-standard physics or astrophysics is the interaction of high-energy protons (see section 3.1), the role of neutrinos in astronomy changes with the transition to high energies - instead of being carriers of information about processes in opaque media, they become markers of interactions of relativistic hadrons whose acceleration to high energies requires the medium to be not too dense.

This transition from neutrinos born in nuclear processes to neutrinos associated with high-energy elementary particle interactions is crucial in distinguishing highenergy neutrino astrophysics as a separate field, to which this review is devoted. Note that one article, even a large one, cannot fully cover all aspects of this actively developing area. This review, therefore, does not pretend to be complete. We will concentrate on astrophysical models of the origin of neutrinos with energies in the $\left(10^{11} \ldots 10^{16}\right) \mathrm{eV}$ range and will therefore focus only on the most relevant experimental results for their study. Beyond the scope of this work are, in particular, ultrahigh energy neutrinos, interesting details of the experimental work on neutrino detection and various results in a certain way related to the study of elementary-particle properties. Even within this framework, a review of the literature will be necessarily incomplete, for which the author apologizes in advance. Books and reviews [1 6] and others, that touch on a variety of aspects of highenergy neutrino astronomy, can be recommended to the reader.

\subsection{High-energy neutrino detection}

a. Neutrino interactions in water. The experimental data to be discussed in this article were obtained using neutrino telescopes that record Cherenkov emission of charged particles, the products of neutrino interactions in large volumes of water (in the solid or liquid state). Interactions of neutrinos with quarks $q$ of nucleons of target atomic nuclei can proceed with the $W$-boson exchange (charged current, CC),

$$
\mathrm{CC}: \nu_{l}+q \rightarrow l+X,
$$

where $\nu_{l}$ and $l$ are neutrinos and charged leptons of the same flavor, $l=e, \mu, \tau$, and $X$ denotes other, hadronic products of the reaction. The other interaction channel is the $Z$-boson exchange (neutral current, $\mathrm{NC}$ ),

$$
\mathrm{NC}: \nu_{l}+q \rightarrow \nu_{l}+X
$$

Similar reactions are possible for antineutrinos.

The probability of interaction between neutrinos and target electrons is low except in the case of the so-called Glashow resonance [7, 8, the direct production of a $W$ boson,

$$
\bar{\nu}_{e}+e \rightarrow W \rightarrow \ldots,
$$

where "..." denotes a well-studied set of $W$-boson decay products, which can include both hadrons and leptons. This process goes only for antineutrinos, for there are no positrons in the target. The resonance occurs at the energy $E_{\bar{\nu}_{e}}=m_{W}^{2} /\left(2 m_{e}\right)$ where $m_{W}$ and $m_{e}$ are the masses of the $W$ boson and electron, respectively.

The result of the interactions observed in the detector depends not only on the type (1), (2), (3), but also on the flavor of the initial neutrino. The CC reaction involving $\nu_{\mu}\left(\bar{\nu}_{\mu}\right)$ results in the production of a relativistic muon, which decay length is, at the energies of interest, usually larger than the size of the detector. The Cherenkov radiation of this single muon is recorded as a narrow track crossing the detector. Note that the widespread notion that such track events are associated only with $\nu_{\mu}$ is not entirely correct: they also include muons from the decay of $\tau$-leptons born in CC interactions of $\nu_{\tau}$, or from $W \rightarrow \mu^{-} \bar{\nu}_{\mu}$ in the case of the Glashow resonance, as well as high-energy $\tau$-leptons that do not have time to decay in the detector (a total of $\sim 10 \%$ of all tracks [9]).

CC events involving $\nu_{e}$ or $\bar{\nu}_{e}$ lead to the formation in the detector of two multiparticle processes, overlapping and forming a common cascade. One of the showers starts with the braking radiation of the electron, whose radiation length in water is only about $36 \mathrm{~cm}$, and the other is associated with the hadronic products of $X$. Note that the longitudinal development of the cascade occurs over a length three orders of magnitude shorter than the length of a muon track (see, e.g., estimates in Ref. [3]).

The CC reaction caused by $\nu_{\tau}$ or $\bar{\nu}_{\tau}$ looks differently in the detector depending on the neutrino energy. The decay length of a $\tau$-lepton with energy $E_{\tau}$ is $\sim 50 \mathrm{~m} \times$ $\left(E_{\tau} / \mathrm{PeV}\right)$, so at $\mathrm{PeV}$ energies such event is observed as a double cascade [10] - at the interaction point, a shower of $X$ hadrons is recorded, and at the decay point - one from $\tau$ decay products, also predominantly hadronic. It is possible to separate the showers both spatially and by the time interval between the two flashes [11. At the energies of $\lesssim \mathrm{PeV}$ these interactions look like normal cascades.

Finally, at NC interactions of neutrinos and antineutrinos of all types only the hadronic cascade is recorded, as the neutrino remaining in the final state leaves the detector without further interactions. 
b. Specifics of observation of track and cascade events. Events interpreted as the Glashow resonance or a double cascade are associated with the highest neutrino energies and thus are very rare 12, 13, so the bulk of astrophysically interesting information is associated with tracks and cascades. In practice, the registration of Cherenkov radiation is made by a three-dimensional array of optical modules with photodetectors viewing a large volume of the target. In order to reduce the background from muons of extensive atmospheric showers (see later in this section), the facility is immersed in water (ice) to a depth of about one kilometer. Photodetectors record the amount of Cherenkov light and, with high accuracy, the moment of the flash. The latter is important because the temporal evolution of the signal makes it possible to determine the direction and speed of a muon or of the cascade development. Illustrations with images of simulated and real recorded events can be found in the reviews cited above, popular science literature (see, e.g., [14]) and the media.

The cascade evolves almost isotropically in the centerof-mass system of the original particles, so in the laboratory frame it looks like an extended "cloud". The neutrino arrival direction is determined considerably worse in this case than for a long linear muon track, see examples below. In contrast, in terms of the energy determination, cascades are indispensable: if a cascade begins in the working volume of the detector (the so-called starting events), then almost all of the energy of the initial neutrino goes to the Cherenkov light and is collected by photodetectors.

For track events, the potential of determining the energy of the initial neutrino is noticeably more modest. The muon track usually extends beyond the boundaries of the facility, so the total recorded energy release in the detector, $E_{\text {dep }}$, gives only a lower bound on the muon energy $E_{\mu}$ at the entrance to the detector, which is estimated using the time evolution of the signal. Since it is not known how much energy the muon has lost before entering the detector, as well as how much of the energy of the original neutrino was transferred to the muon, this measurement allows one to estimate the energy of the muon at birth $\hat{E}_{\mu}$ and the energy of the original neutrino $E_{\nu}$ only in a statistical way. While $E_{\nu}$ is constrained from below quite well, the broad non-Gaussian distribution of possible $E_{\nu}$ corresponding to a given muon detection, extends towards values of $E_{\nu}$ orders of magnitude higher (see Fig. 1). Additionally, note that this statistical distribution, as well as the most likely estimate of $E_{\nu}$, depends on the assumption about the spectrum of astrophysical neutrinos.

c. Arrival directions. Water and ice. In particlephysics terms, the interactions of neutrinos in liquid water and in ice are of course identical, but for the detection of Cherenkov radiation and for event reconstruction, properties of these media are quite different.

Table 1 gives characteristic values of the absorption and scattering lengths of light at wavelengths of the max-

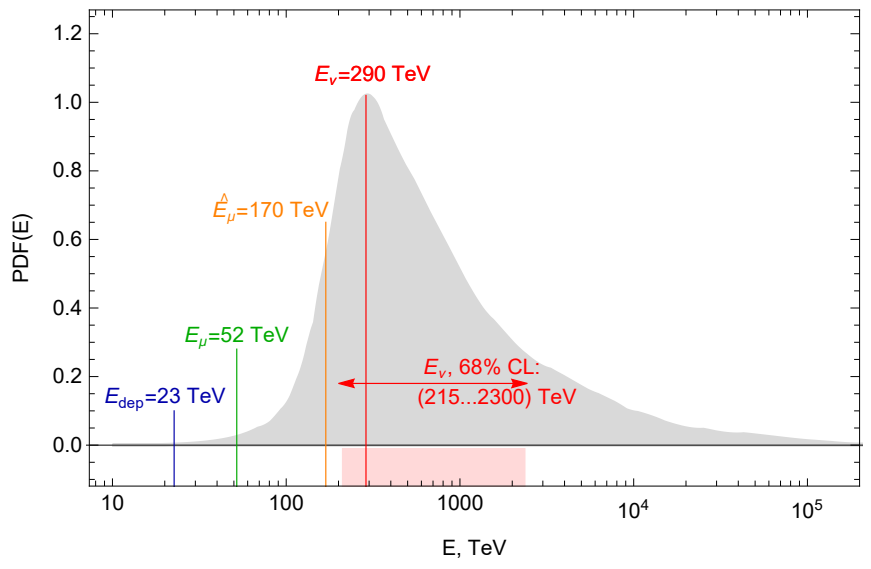

FIG. 1. An illustration of the energy-estimate uncertainty for a track event using the example of one of the best known neutrinos registered by IceCube (IC170922A, coincident with the outburst of the blazar TXS 0506+056, see Sec. 2.3.4. Horizontal axis - energies: the energy deposited in the detector $E_{\text {dep }}=23.7 \pm 2.8 \mathrm{TeV}$, the reconstructed energy of the muon entering the detector $E_{\mu}=52_{-9}^{+11} \mathrm{TeV}$, the energy estimate of the muon at birth $\hat{E}_{\mu} \simeq 170 \mathrm{TeV}$, the most likely neutrino energy $E_{\nu} \simeq 290 \mathrm{TeV}$. The shaded graph shows the probability density function $(\mathrm{PDF})$ of the values of $E_{\nu}$; also shown is the uncertainty region of the $E_{\nu}$ values - from 215 to $2300 \mathrm{TeV}(68 \% \mathrm{CL})$. A power-law spectrum of astrophysical neutrinos with the index of 2.13 is assumed. Plotted on the basis of data from Ref. [15.

imal Cherenkov radiation for typical experimental conditions in different media (for details see Ref. [16]). Under real conditions, these values depend strongly on the particular location, primarily on the depth, varying even within the same installation. Nevertheless, the averaged estimates show that the instruments using liquid water and ice complement each other. In terms of identifying events and measuring their energies, ice is more convenient - it has less illumination from natural sources (bioand chemiluminescence and radioactivity), and the weak absorption allows one to collect more light from each event, lowering the registration threshold and increasing the accuracy of the energy determination. In contrast, for the astrophysical task of identifying neutrino sources, the accuracy of the arrival direction, including both the statistical scatter and systematic errors, is of more importance. The statistical errors are largely determined by the scattering length, so that they are about 5 times smaller in water experiments than in ice experiments; this is true for both cascades and tracks. In the case of cascade events this improvement (from $15^{\circ}-20^{\circ}$ to $3^{\circ}-4^{\circ}$ ) is crucial.

Another important component of the accuracy of the reconstruction of neutrino arrival directions are systematic uncertainties. These come primarily from the accuracy of positioning the detector as a whole and from imperfections in the reconstruction of events, including insufficient knowledge of the properties of the medium. 


\begin{tabular}{cccc}
\hline \hline & $\begin{array}{c}\text { Lake Baikal } \\
\text { water }\end{array}$ & $\begin{array}{c}\text { Salty } \\
\text { water }\end{array}$ & Ice \\
\hline Absorption length, $\mathrm{m}$ & $\sim 20$ & $\sim 50$ & $\sim 100$ \\
Scattering length, $\mathrm{m}$ & $\sim 200$ & $\sim 200$ & $20-40$ \\
Problems & $\begin{array}{c}\sim \text { Shallower available } \\
\text { depth, } \\
\text { bioluminescence, } \\
\text { chemiluminescence }\end{array}$ & $\begin{array}{c}\text { Radioactivity of } \\
\text { dissolved salts, } \\
\text { bioluminescence, } \\
\text { chemiluminescence }\end{array}$ & $\begin{array}{c}\text { Dust inclusions, } \\
\text { clathrates }\end{array}$ \\
\hline \hline
\end{tabular}

TABLE 1. A comparison of water and ice detectors. Longer length corresponds to better optical performance.

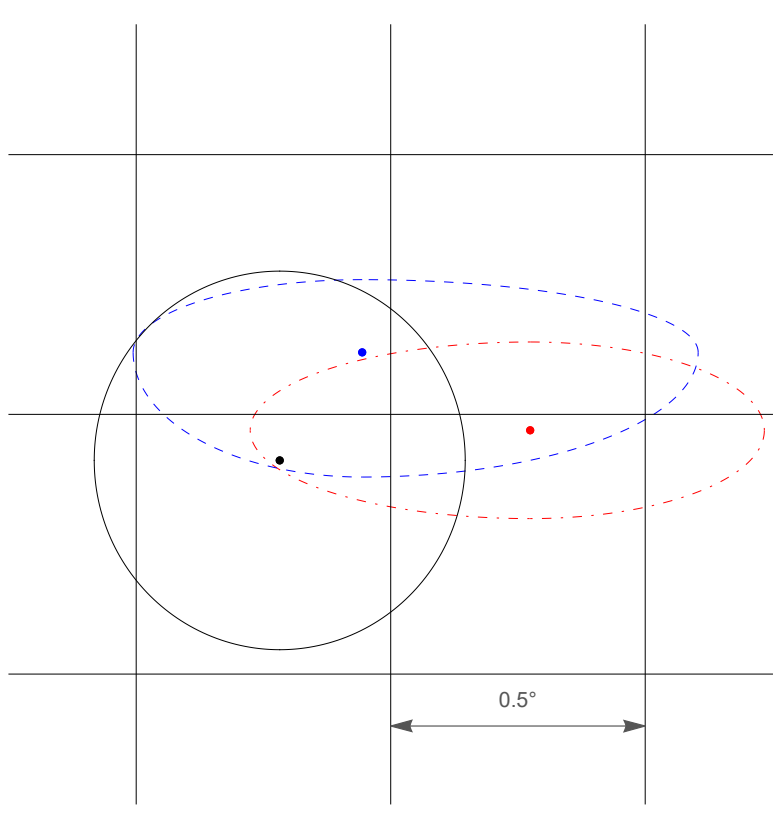

FIG. 2. Illustration of statistical and systematic uncertainties of arrival directions of track events. The arrival directions and their $90 \%$ CL statistical error regions are shown for the same event with energy $\sim 4450 \mathrm{TeV}$, according to Ref. 17. (dashed dashed line), the catalog of alert events 18] (dashed line) and the IceCube 10-year public catalog [19, 20] (solid line). The difference between reconstructions gives an estimate of the systematic uncertainty.

Since inhomogeneities, bubbles and inclusions in water mix, float or settle down, the working volume of a water detector is much more homogeneous and much more uniform and controllable than that of an ice detector. If the accuracy of absolute positioning can be estimated from observation of the shadow of the Moon, the second component of the systematic uncertainties is much harder to estimate. Some estimates of its magnitude may be obtained by comparing the arrival directions of the same events reconstructed with different algorithms and with different ice models, see Figs. 2, 3, where also some typical statistical errors in determination of the neutrino arrival directions are shown.

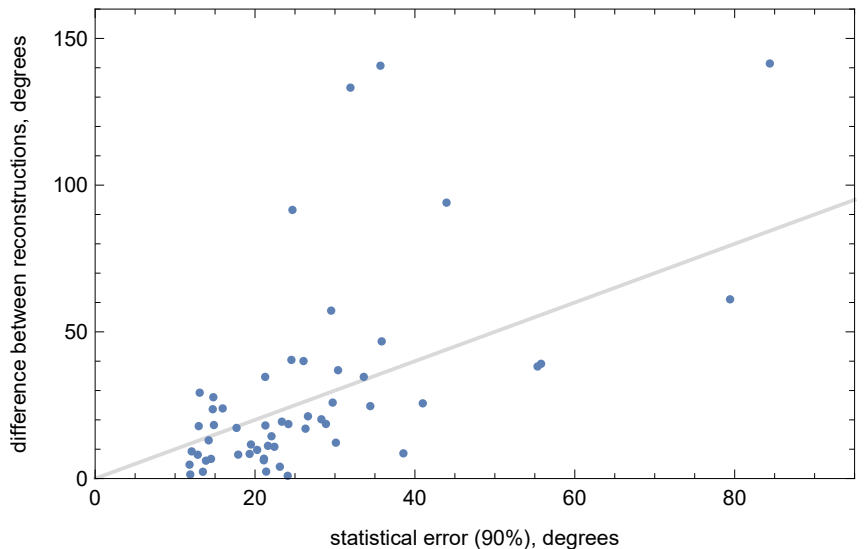

FIG. 3. Illustration of statistical and systematic uncertainties of arrival directions of cascade events. The horizontal axis gives the value of the statistical error (90\% CL) of the arrival direction of HESE cascades of Refs. 21 23, the vertical axis gives the difference between the arrival directions of these events in the original [21-23] and new [24] reconstructions.

d. Passage of neutrinos through the Earth. Neutrino interaction cross sections increase with energy, and at the high energies of interest, the Earth is no longer completely transparent to neutrinos. For different arrival directions, paths through the Earth are different, but the dependence of the interaction probability is not just geometric because the Earth has a very dense core with a sharp boundary. This issue is discussed in more detail in Refs. 1, 3]; here we present only Fig. 4, plotted on the basis of the data from these works. It shows the characteristic neutrino energy, from which the interaction with the Earth is significant, as a function of the zenith angle. Note that some of the interactions are elastic, after which the neutrino continues its motion with lower energy, so that the total flux suppression depends also on the spectrum of the incoming particles, not only on the zenith angle.

e. Atmospheric and astrophysical neutrinos. The main backgrounds for detection of astrophysical neutrinos are muons and neutrinos from interactions of cosmic rays with the Earth's atmosphere. Except that at the highest energies, this background dominates over the signal. For instance, Fig. 5 gives an estimate of the fraction 


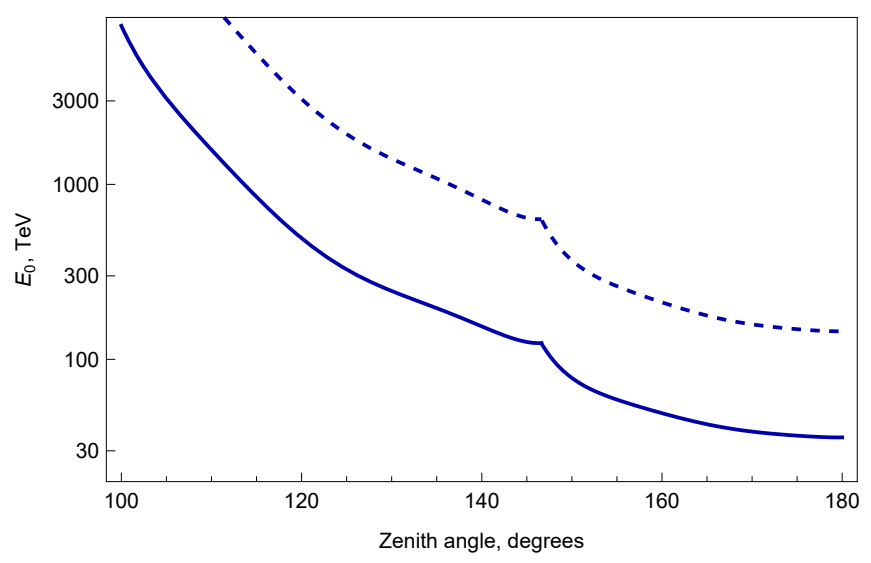

FIG. 4. The critical energy $E_{0}$ for which the optical depth for the electron neutrino with respect to its interaction with the Earth's matter is 1 (solid line) or 2.3 (dashed line, $90 \%$ of neutrinos interact) as a function of the zenith angle. At energies $\gtrsim E_{0}$, the Earth gradually becomes opaque to neutrinos coming from this direction.

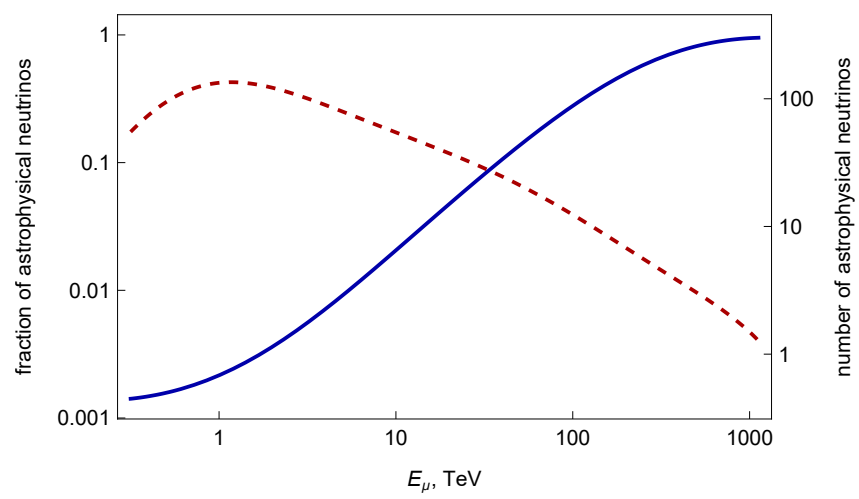

FIG. 5. The expected fraction of astrophysical neutrinos in the total number of muon tracks as a function of energy $E_{\mu}$ (left scale, solid line) and the expected total number of astrophysical neutrinos at a given energy (per 0.1 dex bin in $E_{\mu}$, right scale, dashed line) in a set of $\sim 650000$ IceCube events. Plotted with the data from Ref. 25] (model for 10 years of observations).

of astrophysical events in the set of IceCube muon tracks as a function of the muon energy $E_{\mu}$. Despite such a modest signal-to-background ratio, there are ways to isolate the astrophysical component in the analysis.

For energies of the order of $\mathrm{GeV}$, the process of atmospheric neutrino production in interactions of cosmic rays with hadrons present in the atmosphere is quite similar to the mechanism of the origin of astrophysical neutrinos in $p p$ interactions, see Sec. 3.1 below: $\pi^{ \pm}$mesons are born and then decay, and then the $\mu^{ \pm}$, produced at this first stage, decay as well. The resulting neutrino spectrum reflects the spectrum of cosmic rays, $E^{-2.7}$, and the flavor ratio $\nu_{e}: \nu_{\mu}: \nu_{\tau}$ is 1:2:0. The situation changes for the muon energies $\hat{E}_{\mu} \gtrsim 10 \mathrm{GeV}$, when muons begin to reach to the Earth's surface without decay. As a result, the flavor composition changes so that the ratio of $\nu_{e}: \nu_{\mu}$ becomes $\sim 1: 30$ (electron neutrinos are born in some $K$ meson decays; $\nu_{\tau}$ still has no mechanism to be produced). In addition, at the $\pi$-meson energies $E_{\pi} \gtrsim 100 \mathrm{GeV}$, the latter also have no time to decay - they interact faster with atmospheric hadrons; atmospheric showers begin to develop. Since the decay probability falls with energy as $1 / E_{\pi}$ (Lorentz kinematics), and the interaction probability weakly depends on the energy, in the energy region of interest, the spectrum of atmospheric neutrinos from $\pi$ - and $K$-meson decays follows $\sim E^{-3.7}$ (see Sec. 2.2 below for the discussion of the contribution of charmed hadrons).

The distribution of atmospheric neutrinos in zenith angles peaks strongly for horizontal directions, since in this case the muon's path in the atmosphere is longer, and hence it is more likely to decay. In real analyses at high energies, this dependence is further strengthened by applying muon veto for events coming from above (events with simultaneous signal from other muons from the same shower are rejected), while for $E_{\nu} \gtrsim 50 \mathrm{TeV}$ the Earth's opacity to neutrinos starts to be noticeable for events coming from below. It is the combination of the very soft spectrum $E^{-3.7}$ and the described dependence on the zenith angle which gives the basis for the extraction of the astrophysical signal against the atmospheric background on the statistcal basis, see Figs. 6, 7 in Sec. 2.1 below.

\subsection{Past, present, and future experiments}

The idea of underwater detection of high-energy neutrinos was first proposed by M.A. Markov and I.M. Zheleznykh 26] (see also Ref. 27]). It is not the purpose of this review to discuss in detail the history of neutrino astronomy, nor to give a detailed technical description of the instruments at work, see Refs. [2, 4, 5] and references therein. Brief information about past, present, and emerging detectors, which may be useful in reading the rest of the review, can be found in Table 2 .

\section{KEY EXPERIMENTAL RESULTS}

\subsection{Extraterrestrial origin of neutrinos}

As it has been already noted, we are discussing neutrinos with energies substantially above the nuclear scales. There are no terrestrial sources of such neutrinos (except for narrow beams of neutrinos of accelerator origin, which however do not enter into experimental facilities of the cubic-kilometer scale). The astrophysical signal in the detectors should be separated from atmospheric neutrinos produced by interactions of cosmic rays with the Earth's atmosphere, and from background events caused by muons from the same interactions. When studying a single neutrino event, it is not possible to say definitely 


\begin{tabular}{|c|c|c|c|c|}
\hline Name & Location & Volume, $\mathrm{km}^{3}$ & Years & Note \\
\hline $\begin{array}{l}\text { NT-36, } \\
\text { NT-200, } \\
\text { NT-200+ }\end{array}$ & Baikal & $10^{-4}(*)$ & $1993-2015$ & $\begin{array}{l}\text { First detection } \\
\text { of muon tracks from } \\
\text { atmospheric neutrinos }\end{array}$ \\
\hline AMANDA & South Pole & 0.015 & $1996-2008$ & \multirow{2}{*}{$\begin{array}{l}\text { Atmospheric neutrino } \\
\text { spectrum up to } \sim 100 \mathrm{TeV} \\
\text { and constraints on } \\
\text { astrophysical models } \\
\end{array}$} \\
\hline ANTARES & $\begin{array}{c}\text { Mediterranean } \\
\text { sea } \\
\end{array}$ & $0.025(*)$ & $2006-\ldots$ & \\
\hline IceCube & South Pole & 1.0 & $2006-\ldots$ & $\begin{array}{l}\text { Observation of astrophysical } \\
\text { neutrinos. The largest } \\
\text { statistics }(2021)\end{array}$ \\
\hline Baikal-GVD & Baikal & $\begin{array}{c}0.4(2021)\left(^{*}\right) \\
\geq 1(\text { plan })\end{array}$ & $2016-\ldots$ & \multirow{2}{*}{$\begin{array}{l}\text { Data taking } \\
\text { in progress } \\
\text { of deployment } \\
\end{array}$} \\
\hline KM3NeT & Mediterranean sea & $\sim 1\left(^{*}\right)$, plan & $2019-\ldots$ & \\
\hline IceCube-Gen2 & South Pole & $\sim 10$, plan & project & \\
\hline $\mathrm{P}-\mathrm{ONE}$ & Pacific, Canada & $\sim 3\left(^{*}\right)$, plan & project & \\
\hline
\end{tabular}

TABLE 2. Major experiments in high-energy neutrino astrophysics. Specified are the years of obtaining physical results, including those in incomplete configurations. $(*)$ the volume filled with detecting equipment; water detectors can be used to record high-energy cascades in the volume well in excess of these values.

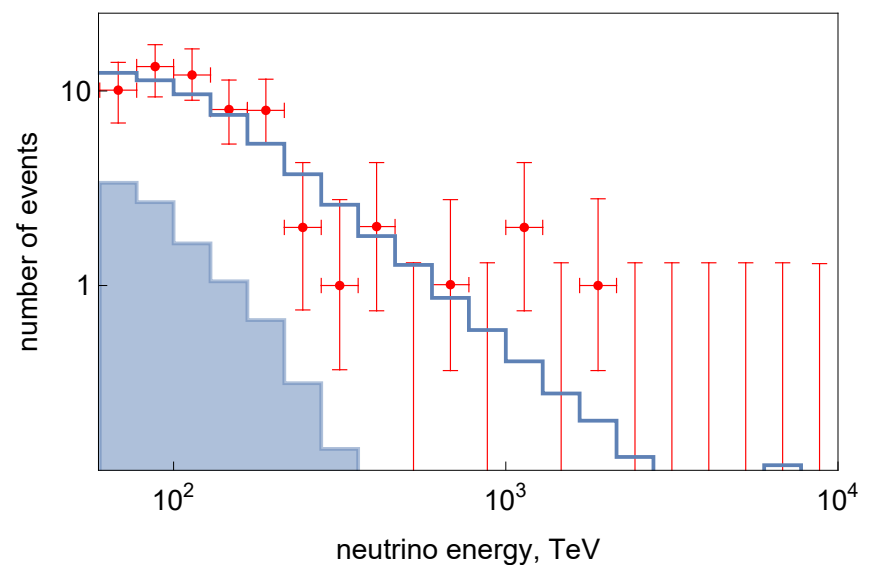

FIG. 6. Distribution of IceCube cascade events, starting in the detector, in the energy deposited in the detector $(E>60 \mathrm{TeV})$. Red dots with error bars - data, dark shading - standard atmospheric background, solid line - the fit of the sum of the background and the astrophysical component. Plotted with the data from Ref. 24].

whether it was of the atmospheric or astrophysical origin, but in examining the ensemble of the data it is possible to distinguish the astrophysical component of the flux against the atmospheric one. For this purpose, one uses the distribution of events in energies (at high energies, hard astrophysical spectra become more pronounced compared to soft atmospheric ones, see Fig. 6) and the zenith angle (see Fig. 7). While astrophysical neutrinos come fairly isotropically (only at the highest energies does the Earth become opaque to them), the distribution of atmospheric neutrinos by zenith angles peaks for hor-

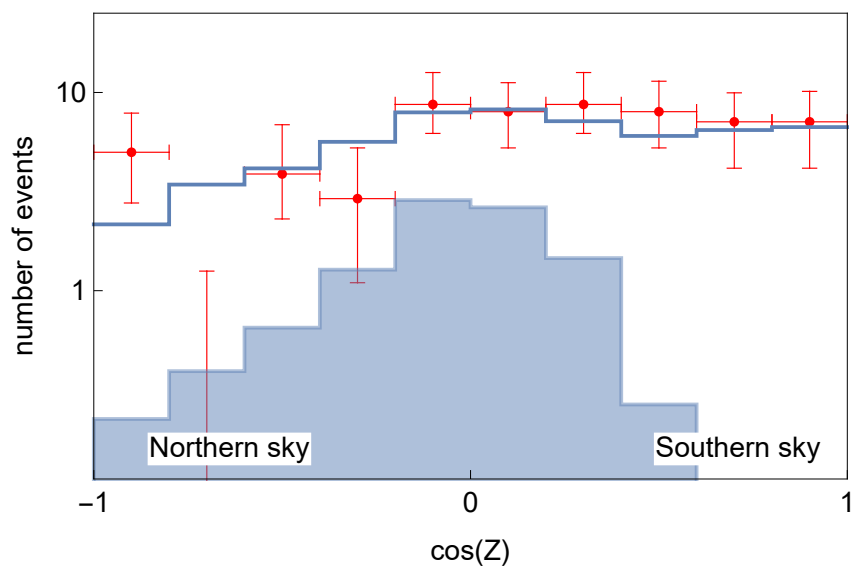

FIG. 7. Distribution of IceCube cascade events $(E>$ $60 \mathrm{TeV})$, starting in the detector, in the zenith angle $Z$. Red dots with error bars - data, dark shading - standard atmospheric background, solid line - the fit of the sum of the background and the astrophysical component. Plotted with the data from Ref. [24].

izontal directions. The atmospheric muons themselves can be filtered out by a simultaneous triggering system mounted on the surface, or - for directions from below simply by the Earth.

Detection of neutrinos of astrophysical origin was first announced by the IceCube Collaboration in 2013 based on the observation of two events with cascades started in the detector with reconstructed energies above $1 \mathrm{PeV}$, for which the atmospheric background is simply negligible. Presently, various analyses based on IceCube datasets with different selection criteria are carried out, and in all 
of them the presence of an astrophysical component of the neutrino flux is established in a statistically significant way, based on a combination of multiple factors. For the most energetic individual events, the probability of their astrophysical origin is determined (we note that for particular samples used in the IceCube analyses, even for those selected according to the most stringent criteria, this probability in average does not exceed $\sim 60 \%$ ).

An independent confirmation of the presence of astrophysical neutrinos by other experiments should be considered as an important step in the development of neutrino astrophysics. Such a result, albeit with low statistical significance, was presented in 2019 by the ANTARES collaboration [28]. The number of events with energies above $100 \mathrm{TeV}$ recorded at the Baikal-GVD facility during data taking in the incomplete configuration is also consistent 29 with the presence of the astrophysical neutrino flux with the parameters measured by IceCube. An eagerly awaited more accurate quantitative verification of the IceCube results will be possible in a few years of Baikal-GVD operation.

\subsection{Spectrum and flavor composition}

Detailed measurements of the spectrum of astrophysical neutrinos, similar to those carried out for cosmic rays, are still difficult because of statistical and systematic uncertainties. The energies of individual track events are estimated with low accuracy which does not allow for any meaningful binning of the spectrum, while the number of reliably studied starting cascade events is small. In addition, the estimation of the astrophysical flux is always based on subtracting the atmospheric background, which is also modeled with uncertainties. As a consequence, most of the spectrum estimates obtained so far use a simple power-law fit,

$$
\frac{d F_{\nu+\bar{\nu}}}{d E_{\nu}}=\Phi_{0}\left(\frac{E_{\nu}}{100 \mathrm{TeV}}\right)^{-\gamma} \times 10^{-18} \mathrm{GeV}^{-1} \mathrm{~cm}^{-2} \mathrm{~s}^{-1} \mathrm{sr}^{-1} \text {, }
$$

where the fit parameters are the normalization $\Phi_{0}$ and the spectral index $\gamma$. The standard parametrization (4) refers to the diffuse isotropic flux of neutrinos and antineutrinos of the same flavor, defined assuming equal fluxes of all six types of neutrinos and antineutrinos (thus, the total flux is obtained by multiplying that given by Eq. (4) by three). Different analyses are more or less sensitive to different energy intervals and different flavors. Parameters of the best-fit spectrum (4), determined in the various IceCube and ANTARES analyses, are given in Table 3. The ranges of energies, which give the main (usually 90\%) contribution to the fit spectrum, are also indicated. A more detailed discussion of the features of the datasets used and of details of the experimental work is beyond the scope of this paper; a rather detailed description of different IceCube analyses is given in a recent paper 24].

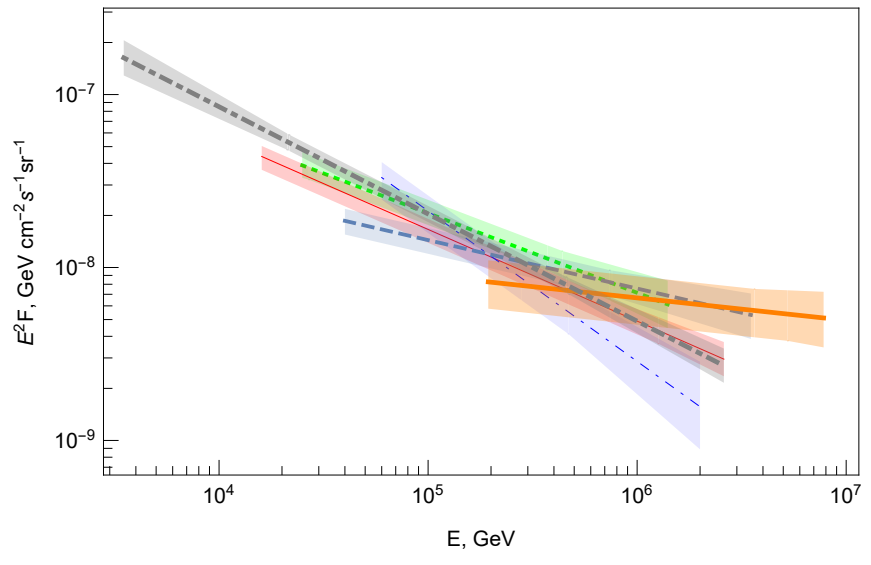

FIG. 8. Astrophysical neutrino spectra (4) from various IceCube analyses (for details and references, see Table 3 . Thick orange solid line $-\nu_{\mu} 2016$; blue dashed line $-\nu_{\mu} 2019$; green dotted line - MESE 2014; thick gray dot-dashed line inelasticity 2018; thin red solid line $-\nu_{e}+\nu_{\tau}$ cascades 2020; thin blue dot-dashed line - HESE 2020. The shading of the corresponding color shows the statistical uncertainties of the corresponding power-law fit.

Although the parameters of the spectra obtained in the different analyses are close to each other in the order of magnitude, their scatter is strikingly greater than the $68 \%$ confidence intervals indicated in the table. Errors of the parameters $\Phi_{0}$ and $\gamma$ are not completely independent, so the contours of the confidence regions in the plane of these two parameters are often compared, see e.g, Ref. 24]. Such a comparison does not always seem to be optimal due to the fact that the energy intervals used in the analyses are different, and the true spectrum is probably different from the exact power law. This latter possibility is supported by a visual comparison of the power-law fitted spectra plotted for the energy ranges of the respective analyses, see Fig. 8. It can be seen that, on average, the spectra become harder at higher energies, and at a fixed energy the agreement between those analyses which have sufficient statistics in this region are not bad. The neutrino fluxes fall rapidly with energy in any case, so the best statistics saturating the fit is obtained closer to the lower boundary of the energy range in use. It is natural to attribute the "measurement" of the power-law index $\gamma$ to the average energy of events contributing to the fit, which is easy to estimate by knowing the $\gamma$ value itself and the energy range. The results of such estimation are shown in Fig. 9. The tendency for the spectrum to become harder with increasing energy is particularly clear in this representation - only one point 24] drops out, which, however, is based on only 60 events, albeit of high quality. Possible reasons for this behavior of the spectrum deserve a more detailed discussion, to which we now turn.

a. Atmospheric background? As discussed above, isolating the contribution of astrophysical neutrinos from the background atmospheric neutrinos at high energies is 


\begin{tabular}{cccc}
\hline \hline Analysis & Energy & $\Phi_{0}$ & $\gamma$ \\
\hline HESE 2020 [24] & $69.4 \mathrm{TeV}-1.9 \mathrm{PeV}$ & $2.12_{-0.54}^{+0.49}$ & $2.87_{-0.19}^{+0.20}$ \\
Cascades $\nu_{e}+\nu_{\tau} 2020[30]$ & $16 \mathrm{TeV}-2.6 \mathrm{PeV}$ & $1.66_{-0.27}^{+0.25}$ & $2.53 \pm 0.07$ \\
MESE 2014 [31] & $25 \mathrm{TeV}-1.4 \mathrm{PeV}$ & $2.06_{-0.3}^{+0.4}$ & $2.46 \pm 0.12$ \\
Inelasticity 2018 [32] & $3.5 \mathrm{TeV}-2.6 \mathrm{PeV}$ & $2.04_{-0.21}^{+0.23}$ & $2.62 \pm 0.07$ \\
$\nu_{\mu} 2016[17]$ & $194 \mathrm{TeV}-7.8 \mathrm{PeV}$ & $0.90_{-0.27}^{+0.30}$ & $2.13 \pm 0.13$ \\
$\nu_{\mu} 2019[25]$ & $40 \mathrm{TeV}-3.5 \mathrm{PeV}$ & $1.44_{-0.24}^{+0.25}$ & $2.28_{-0.09}^{+0.08}$ \\
ANTARES 2019 [28] & & $1.5 \pm 1.0$ & $2.3 \pm 0.4$ \\
\hline \hline
\end{tabular}

TABLE 3. Parameters of the power-law fit (4) spectra of astrophysical neutrinos from various IceCube and preliminary ANTARES analyses.

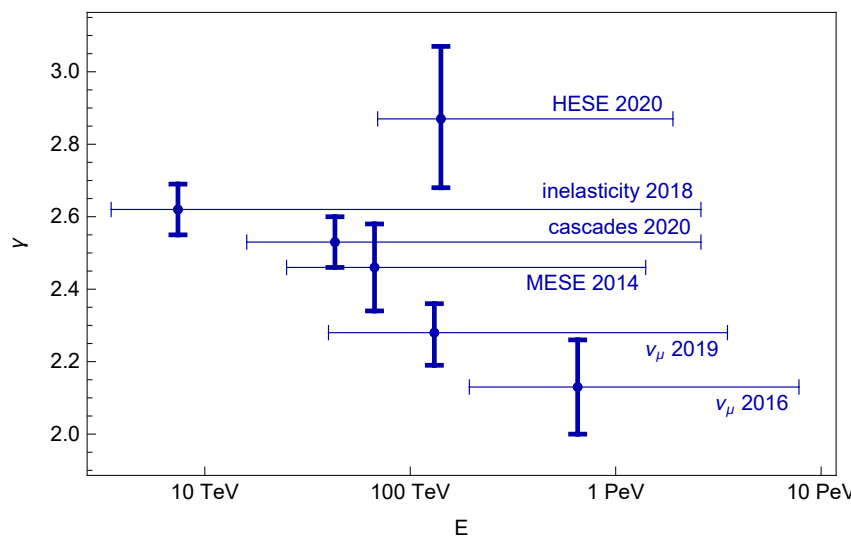

FIG. 9. The index of the power-law fit (4) of the astrophysical neutrino spectrum for various IceCube analyses (for the names of the analyses and references, see Table 3). Bold vertical lines show statistical uncertainties of the reconstructed exponents, and thin horizontal lines show the neutrino energy intervals yielding $90 \%$ of the events for this analysis. The horizontal positions of the dots correspond to the average energy of the events in the data set, estimated from the power-law fit.

quite easy: astrophysical neutrinos have a harder spectrum and a different distribution of zenith angles, see Fig. 6, 7. However, this refers to so-called standard atmospheric neutrinos from decays of $\pi$ and $K$ mesons. At the same time, the products of the interaction of cosmic rays with the atmosphere include charmed mesons, $D_{s}^{ \pm}$, and baryons $\Lambda_{c}$, whose lifetime is about four orders of magnitude shorter. Their decays produce the so-called prompt atmospheric neutrino flux, which has characteristics much closer to those of the astrophysical flux. In particular, due to the short lifetime, the parent hadrons have no time to interact in the atmosphere even at high energies. As as a consequence, prompt neutrinos repeat the spectrum of the initial cosmic rays, $E^{-2.7}$, and are distributed isotropically, up to the effect of passing through the Earth and of event selection. Compared to ordinary atmospheric neutrinos, the prompt flux is substantially enriched in the electron flavor. All of this allowed one to hypothesize 33 35 that only the hard, found in $\nu_{\mu}$,

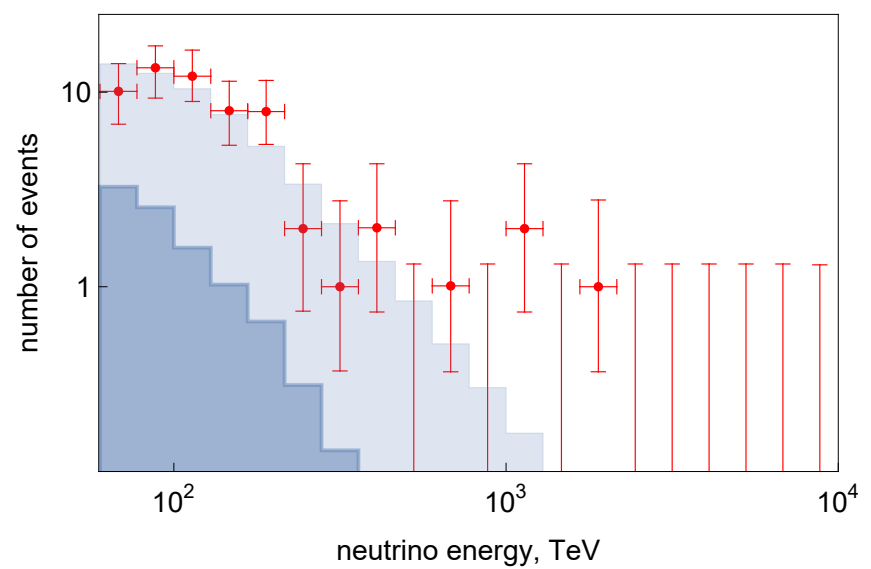

FIG. 10. Distribution of IceCube cascade events, starting in the detector, in the energy deposited in the detector $(E>$ $60 \mathrm{TeV}$ ). Red dots with error bars - data, dark shading standard atmospheric background, light shading - the best fit of the sum of the standard background and the background of prompt atmospheric neutrinos. Plotted with the data from Ref. [24].

component, have the astrophysical origin, while the one derived from the analysis of cascade events, rich in $\nu_{e}$ and having the spectrum closer to $E^{-2.7}$, is explained by prompt atmospheric neutrinos. However, for the cascades arriving from above the horizon, one can perform an additional analysis. The atmospheric neutrinos are not born alone - in the same atmospheric shower, muons are also produced, which can also be detected. The requirement of the lack of simultaneous detection of such muons suppresses the contribution of atmospheric neutrinos coming from above, including prompt ones, and the distribution of the events selected in this way becomes anisotropic. Figs. 10, 11, show the best fit of the observed energy and zenith-angle distributions for the HESE sample assuming a zero astrophysical component - an arbitrary normalization of the prompt neutrino flux is allowed instead. Comparison with Figs. 6, 7 indicates that the spectrum is described by prompt neutrinos equally well as by astrophysical neutrinos, but the zenith-angle distribution excludes this explanation at $>5 \sigma$ confidence 


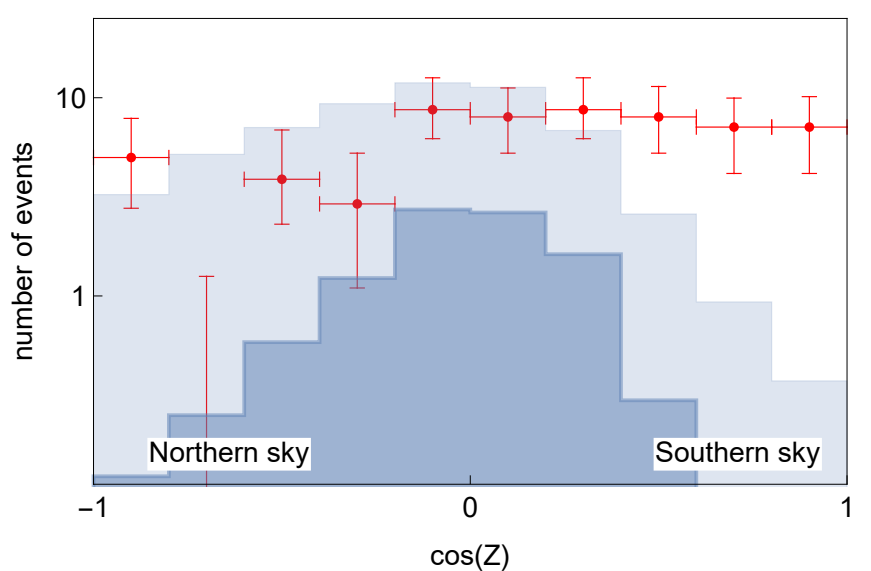

FIG. 11. Distribution of IceCube cascade events, starting in the detector, in the zenith angle $Z$. Red dots with error bars data, dark shading - standard atmospheric background, light shading - the best fit of the sum of the standard background and the background of prompt atmospheric neutrinos. Plotted with the data from Ref. 24].

level. It is worth noting that for a satisfactory explanation of the spectrum, Fig. 10, the prompt flux normalization was increased by a factor of 21.56 as compared to the predictions of modern theoretical models.

It is pertinent to add that an important criterion for the presence of astrophysical neutrinos is $[1,33,36$ the presence of $\nu_{\tau}$ among observed events, since for atmospheric neutrinos, both conventional and prompt, the fraction of $\nu_{\tau}$ is very small. Observations of the first candidates for $\nu_{\tau}$ events 13 support the astrophysical explanation, but the statistics here are still very small.

b. Flavor composition? The spectra (4) with parameters from Table 3 were obtained under the assumption of equality of the fluxes of neutrinos of the three flavors. Since in the analyses based on muon tracks, the atmospheric background is higher due to the presence of both atmospheric neutrinos and atmospheric muons, the spectra obtained from tracks are dominated by higher energies. Such systematics can lead to a dependence of the reconstructed energy on the flux, if in fact this assumption is violated, cf, e.g. 37. In Fig. 12 the ratio of the $\nu_{e}$ and $\nu_{\tau}$ flux to that of $\nu_{\mu}$, calculated in energy bins 30,38 from the power-law fit 25] in the energy range common to the two analyses, is given (deviations of the real spectrum from the power law, discussed below, would lead to a change in this picture). It can be seen that at energies $\gtrsim 200 \mathrm{TeV}$ the results are in a poor agreement with the standard assumption of equality between the flavors; the agreement does improve if one assumes a transition to a different flavor ratio in this energe range. We will see in Sec. 3.1 that this may indeed happen for sources with very strong magnetic fields - so strong that it is not easy, though in principle possible, to reconcile the model with other observational data.

At present, the flavor composition of high-energy neutrinos is not very precisely defined, and available results

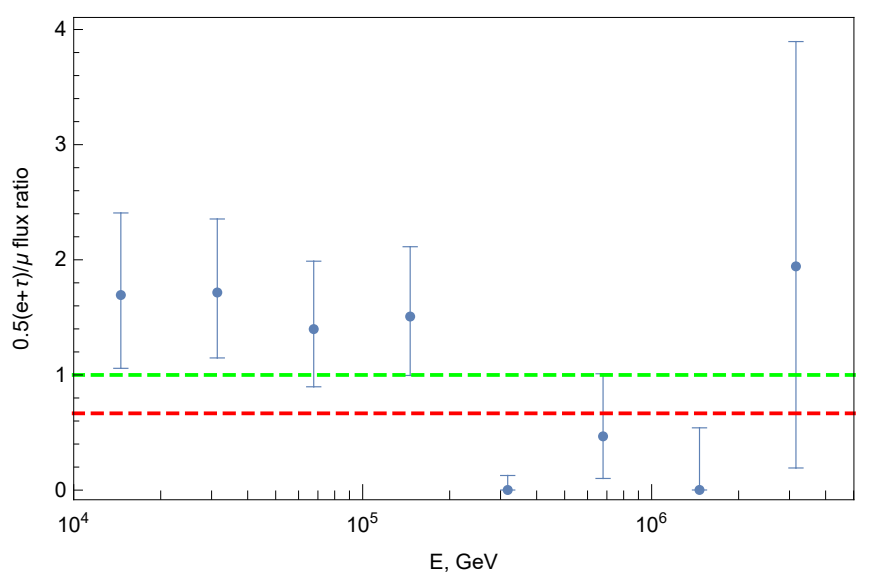

FIG. 12. Ratio of neutrino fluxes of different flavors as a function of energy. Average $\nu_{e}$ and $\nu_{\tau}$ fluxes are taken from the experimental points of the analysis $30,38, \nu_{\mu}$ - from the power-law fit 25. The green dashed line is unity, predicted in normal $\pi$-meson decays, the red dashed line is $2 / 3$, predicted in the muon damp mode (see Sec. 3.1).

and perspectives are discussed in Refs. 39, 40.

c. North-South Anisotropy? Another assumption used in the estimation of the spectra is the isotropy of the diffuse flux. However, none of the approaches to finding astrophysical neutrinos guarantees a uniform sensitivity to the flux coming from different directions. This anisotropy in addition varies with energy, as well as from one analysis to another. Among early attempts to explain the difference in the spectra recovered from cascade and track IceCube events, there were also assumptions about global anisotropy of neutrino arrival directions, see e.g. Ref. 41. Indeed, the track analysis is more sensitive to neutrinos arriving "from below," and at the highest energies - close to the horizon; the cascade analysis covers the entire sky and is sensitive to directions "from above." The presence of a significant anisotropy of the astrophysical flux, e.g, associated with the dominance of a nearby source in the Southern hemisphere, could explain the observed discrepancy in the spectra. To date, no such anisotropy has been detected. The Northern detectors, Baikal-GVD and KM3NeT, could definitively rule out or confirm such an explanation, since for them the notions of "below" and "above" refer to other parts of the sky.

d. Two components of the spectrum? In light of what has been said, it would be natural to simply believe that the spectrum over the entire investigated interval of energies differs from the power law, see Fig. 8 , and the flux at energies of tens of $\mathrm{TeV}$ is higher than expected from the extrapolation of the power law valid above $\sim 200 \mathrm{TeV}$ downward in energies. In Sec. 3, we will see that it is extremely difficult to describe theoretically all the observational data if one assumes the origin of all neutrinos with $\mathrm{TeV}$ to $\mathrm{PeV}$ energies in sources of the same type. The natural next step is to assume two components of the spectrum, one of which (probably ex- 
tragalactic) has a hard spectrum and extends far into the $\mathrm{PeV}$ range, and the other (possibly related to our Galaxy) dominates below $\sim(100 \ldots 200) \mathrm{TeV}$ 37, 42,44. Below we discuss in more detail the observational motivation for such a hypothesis and possible scenarios for the origin of the two components of the diffuse neutrino flux.

\subsection{Arrival directions}

To a large extent, the methods for analyzing arrival directions were inherited by high-energy neutrino astronomy from studies of cosmic rays of ultra-high, $\gtrsim 10^{18} \mathrm{eV}$, energies (see e.g, 45]), whose sources are also unknown. These methods include searches for and constraints on large-scale (of the scale of the entire sky) anisotropy, in particular of that related to the Galaxy's disk or halo, and for deviations from the random distribution of arrival directions at angular distances comparable to the telescope's resolution - namely, the search for autocorrelations, indicating the existence of point sources, and correlations with specific populations of theoretically motivated sources. Unlike for cosmic rays, whose trajectories are deflected by magnetic fields that are often unknown, for neutrinos it is less important to search for mediumscale "spots" on the celestial sphere, but a new possibility of spatiotemporal correlation with flaring sources becomes available.

A general impression of the distribution of neutrino event arrival directions is given by the maps of the sky shown in Fig. 13 (all events, i.e., mostly low energies), and Fig. 14 (highest energies, largest fraction of astrophysical neutrinos). In both cases, no obvious differences from the distribution expected for an isotropic incident flux are detectable. Nevertheless, more subtle analyses, to discussing of which we turn now, allow one to identify or constrain the contributions of various astrophysical sources.

\subsubsection{Constraints on the Galactic anisotropy}

Neutrinos of extragalactic origin are collected from all over the Universe, so their flux must be isotropic to a high degree of accuracy. This distinguishes them from, for example, ultrahigh energy cosmic rays, whose mean free path length is hundreds of megaparsecs, so that their arrival directions could indicate a heterogeneous distribution of sources in that volume (for neutrinos, quantitative estimates of such effect are presented e.g. in Ref. 49). If, however, a significant fraction of the observed neutrinos are produced in our Galaxy, the inhomogeneities in the source distribution should show up in the anisotropy of the arrival directions. Unfortunately, with a significant background of atmospheric events it is difficult to search for such anisotropy; some interesting results have been nevertheless obtained. a. The Galactic plane. The distribution of visible matter in the Galaxy is very different from isotropic stars and gas are mostly contained in the disk visible in the sky as the Milky Way. For a quantitative answer to the question of what fraction of neutrinos may be associated with sources in the disk, the most effective way is to look for "the Milky Way in neutrinos" against a background of uniformly (up to the experimental exposure) distributed atmospheric and extragalactic neutrinos. However, an ambiguity arises here, because the specific distribution of neutrino arrival directions from the disk depends on the assumption about the sources. Often, one uses the models constructed to explain the observed Fermi LAT direction- and energy-dependent intensity of the Galactic diffuse gamma rays. Their free parameters include not only the distribution of matter and magnetic field in the Galaxy, but also the characteristics of the cosmic-ray flux. In particular, the spectrum and composition of cosmic particles, measured in the Solar system, are extrapolated to the entire Galactic disk. Obviously, models of this type should be considered as estimates, and the scatter of their predictions for the neutrino flux demonstrates this systematic uncertainty. Recent observational results rely on the model of Ref. [50], called KRA $\gamma$, and consider two variants of it which assume the cosmic-ray spectrum cutoff either at $5 \mathrm{PeV}$ or at $50 \mathrm{PeV}$ (note that, in general, the results of cosmic-ray studies indicate their probable Galactic origin at higher energies as well). In 2019, from the analysis of the anisotropy of IceCube cascade events [51, an indication was obtained (with the statistical significance of $2 \sigma)$ to the presence of the Galactic component of the neutrino flux with the shape of the spectrum and the spatial distribution described by the KRA $\gamma$ model, with the best-fit normalization of 0.85 (0.65) from the model prediction for cutoffs of $5(50) \mathrm{PeV}$, respectively. The most stringent upper limits (the coefficient of normalization $<1.19$ for the $5 \mathrm{PeV}$ cutoff and $<0.90$ for the $50 \mathrm{PeV}, 90 \% \mathrm{CL}$ ) were obtained from the joint analysis of IceCube and ANTARES [52. As it can be seen from Fig. 15. if the detection of this component is confirmed, it would amount to $\sim 10 \%$ of the IceCube astrophysical flux, in rough agreement with theoretical estimates. However, the mechanism of neutrino production in interactions of cosmic rays with the interstellar medium could be not unique for the Galactic disk; in particular, individual sources accelerating cosmic rays are present there. This analysis does not allow one to constrain their contribution. In this context, a nonparametric comparison of the observed and isotropic distributions of neutrinos in the Galactic latitude looks more universal. It allows one to find or constrain any flux component associated with the Galactic disk (see e.g., [53, 54]).

b. Galactic center - anticenter. Another class of models, including those associated with dark-matter decays or annihilation and with the interactions of cosmic rays in the circumgalactic gas (see Sec. 4.2 below), predicts a different kind of Galactic anisotropy. In this case, 


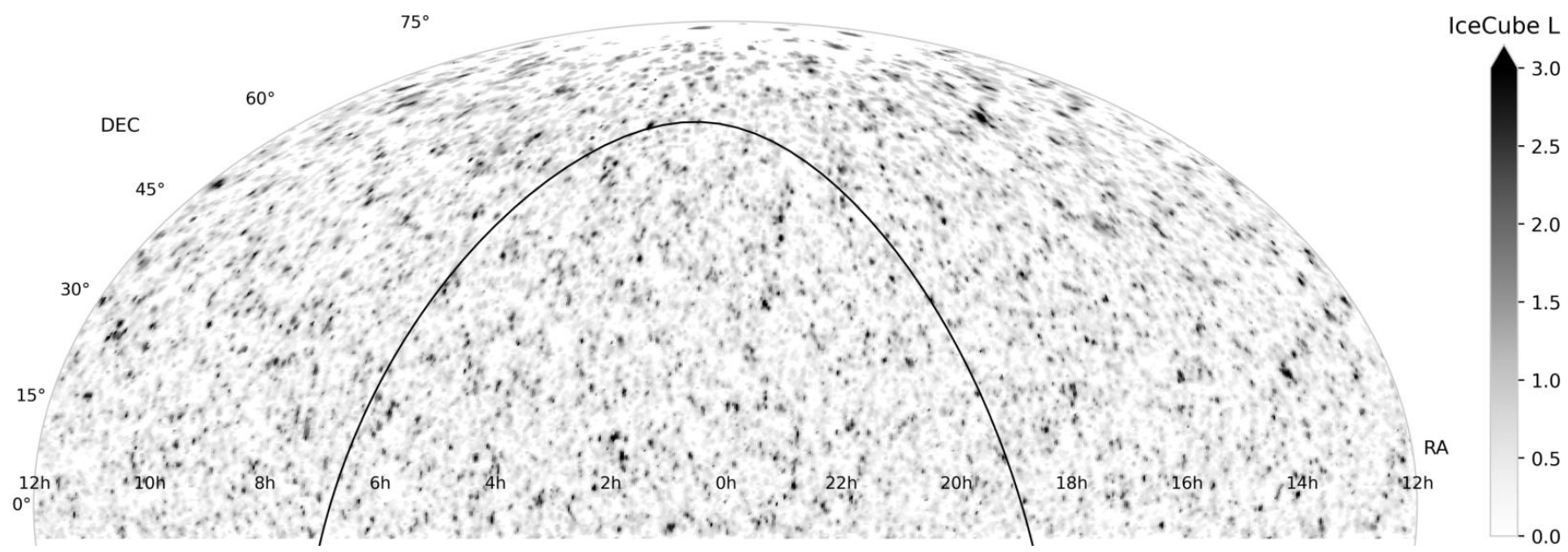

FIG. 13. Weighted distribution of IceCube track-event arrival directions from below the horizon over 7 years of operation (based on [46, 47]). The likelyhood function describing the probability of detecting a source of astrophysical neutrinos in a given direction, taking into account the number of events, the accuracy of determining their arrival directions and the values of the reconstructed energies, is presented. Equatorial coordinates; the Galactic plane is shown by a solid black line. The author is grateful to A. Plavin for his help in preparing this plot.

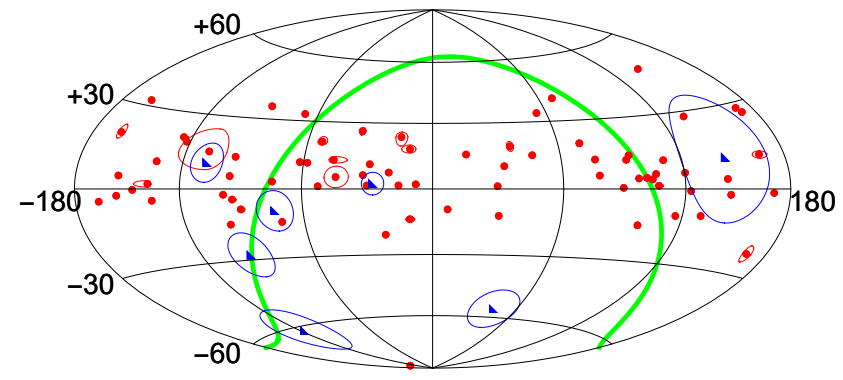

FIG. 14. Distribution of IceCube event arrival directions with published energies above $200 \mathrm{TeV}$, Refs. [17, 18, 21, 24, 48] and GCN and AMON online alerts. The tracks are shown by dots, the cascades are shown by triangles, lines indicate areas of $90 \%$ statistical uncertainty of arrival directions (not always distinguishable for tracks). About half of these events are atmospheric. Equatorial coordinates; the Galactic plane is shown as a solid bold line.

the sources are not concentrated in the disk, but are distributed in the Galaxy in a more or less spherically symmetric way, with concentration decreasing from the Galactic center down to the virial radius, $\gtrsim 200 \mathrm{kpc}$. Together with the non-central position of the Sun in the Galaxy, this leads to the dipole-like anisotropy of arrival directions with the maximum of the flux from the center of the Galaxy and the minimum from the opposite direction 55. It is also convenient to search for such anisotropy, sensitive to the details of the source distribution, in the data with nonparametric tests 54. Recently, experimental data analyses have used this approach in the context of particular dark-matter models [56, 57] together with the analysis of the contribution of dark matter to the observed neutrino spectrum [58, 59. Increas-

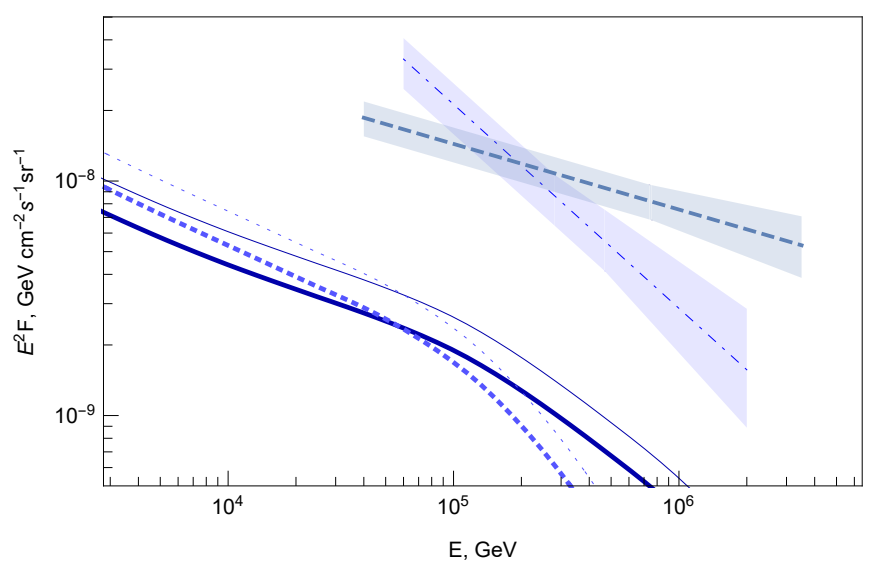

FIG. 15. Neutrinos from the Galactic plane assuming proportionality of the flux to the predictions of the $\mathrm{KRA} \gamma$ model (in terms of one-flavor flux $\nu+\bar{\nu}$ ). Bold curves - the best fit from IceCube cascade events 51, thin curves - the strongest upper limits (90\% CL) from the joint analysis of IceCube and ANTARES [52] (solid lines - KRA $\gamma$ with cosmic-ray cutoff at $50 \mathrm{PeV}$, dotted - at $5 \mathrm{PeV}$ ). The lines with the shaded uncertainty region show the total astrophysical neutrino fluxes from IceCube data (blue dashed line $-\nu_{\mu} 2019$ [25]; thin blue dot-dashed line - HESE 2020 24).

ing the sensitivity to particular models, these studies inevitably lose in universality. In most cases, adding a contribution with such anisotropy leads to an improvement in the description of the data, but this improvement is not statistically significant.

c. Large Galactic structures. The distribution of gas in the Galaxy and in the circumgalactic space is heterogeneous. In terms of the models of the neutrino origin discussed in the literature, one singles out so-called 


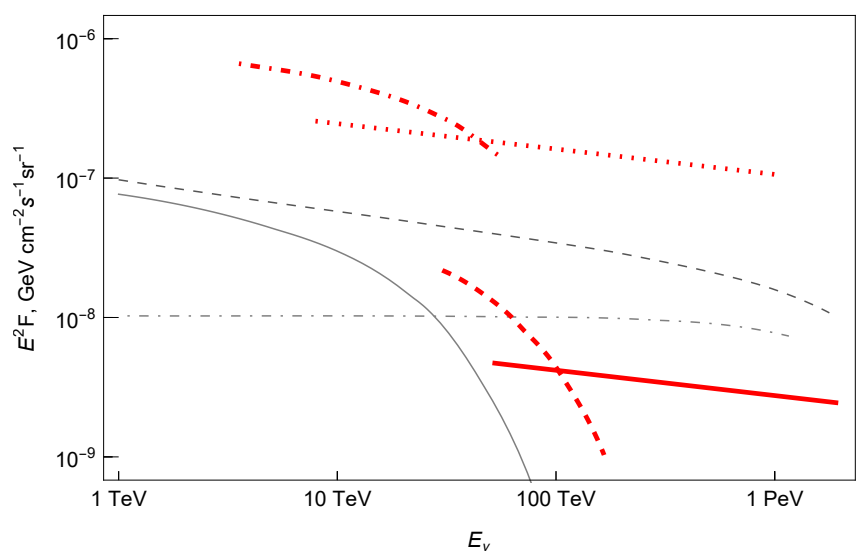

FIG. 16. Constraints on models of neutrinos from Fermi bubbles. Bold red - upper limits on the neutrino flux $(\nu+\bar{\nu}$, one flavor, under the $E^{-2.18}$ spectrum assumption) from ANTARES 61] (dot-dashed line - spectral cutoff at $50 \mathrm{TeV}$, dashed line - no cutoff) and IceCube 46] (dashed line - spectral cutoff at $50 \mathrm{TeV}$, solid line - no cutoff). Thin gray lines - proposed models (dashed line - 62, dot-dashed line - leptohadronic 63 and solid - hadronic 63 ).

Fermi bubbles, large-scale formations above and below the Galactic disk in its central region 60, possibly related to the past activity of the Galactic nucleus. Their contribution to neutrino fluxes can be constrained by treating them as extended sources, to which a higher flux of diffuse radiation in the sky map corresponds. The results of such analyses did not reveal any excess of neutrinos from the Fermi bubbles and allowed one to rule out some models, see Fig. 16 .

A particular prediction of anisotropy arises also for the model of the Local Bubble (see Sec. 4.2), inside which the Solar system is located. The arrival directions of neutrino events in this model should concentrate in spots whose positions are determined by the direction of the Galactic magnetic field lines in the vicinity of the Sun. Because of great uncertainty in the knowledge of the Galactic magnetic field and of difficulties in quantitative analysis, the search for such anisotropy remains an interesting task for future.

\subsection{2. "Blind" search for point sources}

Given the considerable uncertainty regarding theoretically motivated high-energy neutrino sources, the socalled blind search for their possible sources is becoming popular. Within this approach, one searches the entire sky for clusters of neutrinos coming from close directions that could indicate the most powerful point sources. The modern version implements this by constructing the maximum likelihood function, which takes into account not only the arrival directions, but also the accuracy of their determination, which differs from event to event, as well as the energies: a cluster of even a few neutrino events al- lows one to estimate the hypothetical neutrino spectrum from a given direction, and if it is harder than the atmospheric one, then the probability that we are dealing with a real source and not with a background fluctuation is higher. Based on such maps, of which Fig. 13 is an example, many of the results of this and the next subsections have been obtained.

A significant disadvantage of the blind approach is that only the most powerful sources could be revealed in this way with high statistical significance. Indeed, in analyzing each individual direction, it is possible to determine the statistical probability that the cluster of events with all its characteristics arises as a result of a random fluctuation. To do this, it is sufficient to run a significant number of simulations of random sets of events and to calculate how often the observed, or larger, value of the likelihood function is achieved due to fluctuations in these random sets. This probability ${ }^{1}$ is the main characteristic of the significance of finding a hypothetical source. However, here we should distinguish between the probabilities of accidentally finding a "source" (1) in a given predetermined direction and (2) anywhere in the sky. In the blind source search problem, we are dealing with the case (2). The corresponding probability is much larger, which means that even a pronounced cluster of arrival directions can easily turn out to be random. If the probability of a random match for a given direction (pre-trial) is $p_{1}$, then for $N$ independent attempts, the probability (post-trial) becomes $p \sim N p_{1}$. If the assumption of independence of attempts is violated, $p$ is determined from a Monte-Carlo simulation. The ratio $p / p_{1}$, called the penalty factor (see e.g., 64]), for a blind search of neutrino sources is easy to estimate approximately. Assume that the area of the uncertainty of the arrival direction of a track event is of the order of 2 square degrees, and half of the celestial sphere, that is, $2 \pi$ steradian, is involved in the analysis. Then we find the number of quasi-independent directions as the ratio of these areas, $\sim 10^{4}$ (a more correct estimate is required to take into account the anisotropy of the exposure). This means that to assert a $3 \sigma$ statistical significance of a source found in a blind search (i.e., $p \sim 10^{-3}$ ), one would need to have $p_{1} \sim 10^{-7}$, that is, the pre-trial significance $>5 \sigma$.

The most significant blind-search result for the dataset with the largest exposure, 10 years of IceCube track events, corresponds to the direction in the sky, which is $0.35^{\circ}$ away from the galaxy NGC 1068, a nearby galaxy with intense star formation. For this direction, $p_{1}=3.5 \times 10^{-7}, p=9.9 \times 10^{-2}$, which corresponds to the post-trial significance of $\sim 1.6 \sigma$. Additionally it

\footnotetext{
${ }^{1}$ For convenience, it is often recalculated into standard deviations for a normal distribution and is said to be significant at a certain amount of $\sigma$. Although we will also follow this tradition in this article, it is worth remembering that only the probability value itself is meaningful, since all involved distributions tend to be non-Gaussian.
} 
should be noted that the fit indicates a very soft neutrino spectrum from this direction: the power-law index of the spectrum is 3.4, consistent with that expected for atmospheric, rather than astrophysical, neutrinos. Thus, at the time of writing this review, no individual source in the sky has been detected in a blind search with post-trial significance exceeding even $2 \sigma$.

A way to increase the sensitivity of such an analysis to weaker sources is to use a priori fixed catalogs of theoretically motivated neutrino sources. If the sources in the catalog are of one and the same type, then we are talking about a population analysis, and the cumulative signal from many, even weak, sources can be registered by summing multiple signals from their directions, each of which is statistically insignificant. This will be discussed in Sec. 2.3.3. Here we mention recent attempts to increase the sensitivity of the blind search by combining different types of sources. A catalog of specific sources whose neutrino origins have been discussed in the literature is compiled and supplemented by the sources somewhat similar to them. A wide variety of objects fall into the same list. A blind search is limited to the directions of these "motivated" sources, and the penalty factor becomes equal to the number of sources in the catalog, i.e. instead of $\sim 10^{4}$ it becomes, for example, $\sim 10^{2}$. The danger of this approach is that some of the sources were actually "motivated" by previous, based on partial neutrino samples, analyses, which revealed excesses of neutrino events from their directions. Such an analysis can only make astrophysical sense if along with the answer to the question, from which sources the excesses of neutrino events are seen, compared to random, there will also be an answer why they are not seen from other similar sources.

Nevertheless, even along this path, no single source with a post-trial significance $>3 \sigma$ was found. Ref. 65 analyzed a catalog of 110 gamma-ray sources identified from observational data in different bands, based on various criteria. This included 12 sources in the Galaxy, 3 nearby galaxies - the Large and Small Magellanic Clouds and the Andromeda galaxy, M31, 4 starburst galaxies, 89 active galactic nuclei and 2 unidentified sources. All sources were studied as point sources, although the angular sizes of some Galactic sources, and especially of nearby galaxies, are noticeably larger than the IceCube angular resolution for tracks. For two sources the posttrial significance was $>2 \sigma$, they are the already mentioned galaxy NGC 1068 and the blazar TXS 0506+056, which will be discussed in more detail in Sec. 2.3.4. Additionally, in Ref. 65 it has been observed that another two other sources (blazars PKS $1424+240$ and GB6 1542+6129) have pre-trial $p_{1}<2 \times 10^{-3}$ and that the presence of 4 sources out of $\sim 100$ with such low $p_{1}$ is itself unlikely for a random fluctuation. The statistical analysis indicated a probability of such a of coincidence $\sim 5 \times 10^{-4}$ (significance $\left.\sim 3.3 \sigma\right)$. This result, however, does not take into account the aforementioned arbitrariness in the compilation of the source catalog; in particular, the removal from it of TXS $0506+056$, widely discussed previously, reduces the significance to $2.3 \sigma$ [65]. The authors of Ref. 65 conclude from this analysis that the data indicate the presence of real sources in their catalog; however an astrophysical interpretation of this result is difficult.

So far we have mainly referred to the results of the search for neutrino sources in the Northern sky from IceCube track events. Similar work has been done for the Southern sky in the joint analysis of IceCube and ANTARES 66 and also did not result in the detection of statistically significant individual sources, including those in the region of the Galactic Center.

The lack of manifestations of significant individual sources in the analysis described in this section indicates that the observed flux of astrophysical neutrinos is produced by a large number of not very strong sources. This fact can be used to constrain the source models, see Sec. 3.2 below.

\subsubsection{Source populations}

The next natural step after a blind search is to take a population of the same type of astrophysical objects that are potential neutrino sources, and check whether the entire population has an excess of arrival directions compared to a random distribution. In this way one can find or constrain the coherent effect from many sources of the same class, each of which is not strong enough to be detected in a statistically significant way in an individual or blind analysis. Technically, such an analysis can be performed either using the likelihood function described in Sec. 2.3.2 (test statistics are based on the sum of the values of the likelihood function for all directions from the sources in the catalog), or by counting the number of sources from the catalog in the area near each neutrino arrival direction (in this case, the test statistics is simply the total number of "source-neutrino pairs"). In both cases, a weight can be assigned to each source, e.g., depending on the flux of its electromagnetic radiation in a particular band or from other considerations. In the second approach, the size and the shape of the region are given by the accuracy of determination of the neutrino arrival direction (e.g., at the $90 \%$ CL). These approaches have their pros and cons: the likelihood function allows one to account for $10 \%$ of events that fall outside the $90 \%$ CL angular resolution, and may contain additional information, encoded in continuous characteristics of events, such as energy, influencing the probability that a given neutrino is astrophysical. At the same time, when counting events, it is easier to account for the poorly known systematic error in determination of the arrival direction of neutrino, discussed in Sec. 1.2 (see e.g., 67, 68). Typically, the likelihood function is used for large numbers of neutrino events (low energies) or for extended uncertainty regions of complex shape (cascades).

Table 4 provides a summary of numerous (perhaps not 
all) published analyses of correlations of neutrino arrival directions with potential source populations. As one can see, there are only a few statistically significant results. The first fact that draws attention is the lack of correlations with gamma-ray bright blazars from the Fermi LAT catalogs, which, before the detailed analysis of IceCube results, were considered to be among the most likely sources of high-energy neutrinos. That said, a few statistically significant results point to blasars selected according to other criteria. Let us briefly discuss them.

By definition, a blazar is a nucleus of an active galaxy that has a relativistic jet pointing almost exactly at the observer. Relativistic kinematic effects lead in this case to strong amplification of the observed radiation flux. The same mechanism of amplification works for neutrinos. Physical conditions in nuclei and jets allow protons to be accelerated to the energies required for neutrino production. All of this together makes blazars very attractive candidates to high-energy neutrino sources.

While identification as a blazar is determined only by this geometric characteristic of the jet, physical conditions in these sources may be different. They are manifested primarily in in the bands where the blazar emits the dominant part of its non-thermal radiation. The main characteristic here is the frequency, corresponding to the maximum of the synchrotron peak in the broadband spectral energy distribution, which varies from $\nu_{\text {peak }}<10^{12} \mathrm{~Hz}$ (radio band - radio quasars with a flat spectrum, FSRQ) to $\nu_{\text {peak }}>10^{17} \mathrm{~Hz}$ (X-ray band - extreme BL Lac type objects, HBL). In many studies, the primary focus has been on blazars bright in the gamma-ray band, observed by Fermi LAT (1-100 GeV). These are the highest energies at which fairly uniform over the sky samples of sources are available; in addition, at even higher energies, the Universe becomes not completely transparent to gamma rays because of $e^{+} e^{-}$ pair production, see Sec. 3.1. Unfortunately, due to the insufficient sensitivity of Fermi LAT and the strong variability of blasars in the gamma-ray band, the samples of gamma-ray bright sources capture only a small fraction of blazars. It is also possible that this fraction is unrepresentative because the origin of gamma rays in different classes of blazars can be very different. The universal criterion for the presence of a relativistic jet directed towards us is the observation of a compact (no more than a few parsecs in size) region of intense radiation at the center of the source, located at a gigaparsec-scale distance [87, 88]. For this one requires the angular resolution, achievable in modern astronomy only with very-long baseline radio interferometry (VLBI) 89. A catalog of such compact, VLBI-selected, objects, the Radio Fundamental Catalog (RFC), was the basis for the search for neutrino arrival-direction coincidences with blazars in Refs. 67, 77]. Specifically, a complete, limited in the compact-component flux at $8 \mathrm{GHz}$, sample of about 3400 blazars isotropically distributed over the celestial sphere, was used.

Ref. 67 analyzed a sample of 56 arrival directions of published IceCube track events with neutrino energies $E_{\nu}>200 \mathrm{TeV}$. The VLBI flux of complete-sample sources near the neutrino arrival directions (more precisely, in the $90 \%$ CL regions of statistical uncertainty of the arrival directions plus the systematic uncertainty estimated in the analysis) was used as the test statistics. This average flux turned out to be significantly higher than expected for the random distribution of arrival directions of neutrino events. After accounting for the penalty factors associated with variation in the amount of the systematic error, the statistical significance of the established relationship between VLBI sources and highenergy neutrinos was found to be $3.0 \sigma$. Additionally, Refs. 67, 90, found the coincidence of neutrino arrival times with radio flares of these correlated blazars, see Sec. 2.3.4.

In the spring of 2020, the IceCube collaboration published the values of the likelyhood function described in Sec. 2.3.2, constructed from seven years of track-event observations (this is the one presented in Fig. 13). In Ref. [77, based on these data (for directions from below the horizon, where the flux of atmospheric muons is strongly suppressed), the correlation of directions with the maximum probability of locating a point source of astrophysical neutrinos with directions to blazars from the same RFC catalog was established (significance $3.1 \sigma$ post trial). The joint statistical significance of the observations 67] and [77] was estimated as they were based on the same sample of sources. To make the two analyses completely independent, directions of neutrinos with energies above $200 \mathrm{TeV}$, used in the analysis 67. and recorded during the time period for which the likelyhood map was constructed, were cut from the map. The cumulative statistical significance of neutrino correlations with the VLBI-selected blazars was found to be $4.1 \sigma$. The data underlying the likelihood function were dominated by events with energies from a few $\mathrm{TeV}$ to several tens of $\mathrm{TeV}$, and the unexpected result of this analysis was the association of neutrinos of the entire range of energies studied by IceCube, from $\mathrm{TeV}$ to $\mathrm{PeV}$, with sources of the same class. The sources in the complete sample included in the analysis are responsible for about $25 \%$ of the astrophysical flux estimated from the muon tracks (assuming a power law (4) with parameters 25] over the entire energy range). Taking into account the correction for similar sources not present in the catalog raises this value to $\sim 100 \%$.

It should be noted that in Ref. [78, the same set of VLBI-bright blazars was used for a correlation analysis with the recently published 10 -year IceCube public data set of muon tracks 19, 20. Despite the increased, compared to the 7-year set [46, 47] used in Ref. [77, exposure, this analysis found no significant correlations. The contribution of blazars from the catalog to the flux of astrophysical neutrinos was limited to $\lesssim 30 \%$, which is consistent with the result of Ref. [77] $(\sim 25 \%)$. Note that this IceCube dataset is not well suited to test the hypotheses formulated earlier, since it contains neither 


\begin{tabular}{|c|c|c|c|c|c|c|}
\hline $\begin{array}{l}\text { Source } \\
\text { sample }\end{array}$ & $\begin{array}{c}\text { Number } \\
\text { of sources }\end{array}$ & $\begin{array}{l}\text { Neutrino } \\
\text { sample }\end{array}$ & Method & Reference & $\begin{array}{c}\text { Post-trial } \\
\text { significance }\end{array}$ & $\begin{array}{c}\text { Contribution } \\
\text { to the flux }\end{array}$ \\
\hline \multicolumn{7}{|c|}{ Galactic sources } \\
\hline Supernova remnants & 23 & I $\nu_{\mu}(10)$ & $\mathrm{L}$ & 65 & - & \\
\hline Pulsar wind nebulae & 33 & I $\nu_{\mu}(10)$ & $\mathrm{L}$ & 65 & - & \\
\hline Pulsar wind nebulae & 35 & I $\nu_{\mu}(9.5)$ & $\mathrm{L}$ & 69 & - & $<48 \% \nu_{\mu}$ \\
\hline $\begin{array}{c}\text { Unidentified gamma-ray } \\
\text { sources }>100 \mathrm{GeV}\end{array}$ & 23 & I $\nu_{\mu}(10)$ & $\mathrm{L}$ & 65 & - & \\
\hline
\end{tabular}

Extragalactic sources other than blazars

\begin{tabular}{|c|c|c|c|c|c|c|}
\hline Starburst galaxies & 45 & I $\nu_{\mu}$ & $\mathrm{L}$ & 70 & - & \\
\hline Starburst galaxies & 64 & A $(10)$ & $\mathrm{L}$ & 71 & - & \\
\hline Radio galaxies & 63 & A $(10)$ & $\mathrm{L}$ & 71 & - & \\
\hline Dust-obscured galaxies & 15 & A $(10)$ & $\mathrm{L}$ & 71 & - & \\
\hline $\begin{array}{l}\text { Gamma-bright (4LAC) } \\
\text { AGN other than blazars }\end{array}$ & 65 & I $\nu_{\mu}$ & $\mathrm{L}$ & 70 & - & \\
\hline $\begin{array}{c}\text { Large-scale extra- } \\
\text { galactic jets }\end{array}$ & 94 & I HE all & $\mathrm{C}$ & 72 & - & \\
\hline \multicolumn{7}{|c|}{ Gamma-selected blazars } \\
\hline 3FGL & 729 & I $\nu_{\mu} 200+$ & $\mathrm{C}$ & 73 & - & \\
\hline 2FHL BL Lacs & & I $\nu_{\mu} 200+$ & $\mathrm{C}$ & 74 & - & \\
\hline 2FHL HBL & 149 & I $\nu_{\mu}(7)$ & $\mathrm{L}$ & 48 & - & $<27 \% \nu_{\mu}$ \\
\hline 2FHL HBL & 149 & I HE all & $\mathrm{C}$ & 75 & - & \\
\hline $3 \mathrm{FHL}$ & 1301 & I $\nu_{\mu}$ & $\mathrm{L}$ & 76 & - & $<17 \% \nu_{\mu}$ \\
\hline 3FHL & 1301 & I HE $\nu_{\mu}$ & $\mathrm{C}$ & 68 & - & \\
\hline 3LAC HBL & 386 & I HE $\nu_{\mu}$ & $\mathrm{C}$ & 75 & - & \\
\hline $3 \mathrm{LAC}$ & 1255 & A $(10)$ & $\mathrm{L}$ & 71 & - & \\
\hline 3LAC FSRQ & 414 & I $\nu_{\mu}(7)$ & $\mathrm{L}$ & 48 & - & \\
\hline $4 \mathrm{LAC}$ & 2796 & I $\nu_{\mu}(3)$ & $\mathrm{L}$ & 70 & - & $\lesssim 11 \% \nu_{e, \tau}$ \\
\hline $4 \mathrm{LAC}$ & 2794 & I HE $\nu_{\mu}$ & $\mathrm{C}$ & 68 & - & \\
\hline $\mathrm{VOU} \gamma \mathrm{HBL}$ & & $\mathrm{I} \mathrm{HE} \nu_{\mu}$ & $\mathrm{C}$ & 68 & $3.2 \sigma$ & $\sim 21 \% \nu_{\mu}$ \\
\hline \multicolumn{7}{|c|}{ Blazars selected by other criteria } \\
\hline 2WHSP & 1681 & I $\nu_{\mu}(7)$ & $\mathrm{L}$ & 48 & - & \\
\hline 2WHSP & 1681 & I HE all & $\mathrm{C}$ & 75 & - & \\
\hline $3 \mathrm{HSP}$ & 2011 & $\mathrm{I} \mathrm{HE} \nu_{\mu}$ & $\mathrm{C}$ & 68 & $2.8 \sigma$ & \\
\hline $\mathrm{RFC}$ & 3388 & I $\nu_{\mu} 200+$ & $\mathrm{C}$ & 67 & $3.1 \sigma\}_{41 \sigma}$ & \\
\hline $\mathrm{RFC}$ & 3411 & I $\nu_{\mu}(7)$ & $\mathrm{L}$ & 77 & $3.0 \sigma \int^{1.10}$ & $\sim 25 \% \nu_{\mu}$ \\
\hline $\mathrm{RFC}$ & 3388 & I $\nu_{\mu}(10)$ & $\mathrm{L}$ & 78 & - & $\lesssim 30 \% \nu_{\mu}$ \\
\hline
\end{tabular}

TABLE 4. Results of searches for correlations of high-energy neutrino arrival directions with populations of astrophysical sources. Source samples: all Galactic sources are selected from observations above $100 \mathrm{GeV}$; for the selection of other gamma-ray sources, Fermi LAT catalogs were used: all sources, 3FGL [79]; sources detected above $50 \mathrm{GeV}, 2 \mathrm{FHL}$ [80], and above 10 GeV, 3FHL [81; gamma-ray bright active galactic nuclei (AGN), 3LAC 82] and 4LAC 83, - and the VOU-blazars database 84]. Non-gamma-ray related selection criteria for blazars included a high-frequency $\left(>10^{15} \mathrm{~Hz}, \mathrm{HBL}\right)$ synchrotron peak, 2WHSP [85] and 3HSP 86 catalogs, and the presence of a compact core visible with very-long baseline radio interferometers, RFC catalog (http://astrogeo.org/rfc). Neutrino samples: based on the data from IceCube (I) or ANTARES (A), $\nu_{\mu}-$ only track events, number in parentheses - number of years of exposure, HE - published individual high energy events, 200+ - energies above $200 \mathrm{TeV}$. Method: L - likelihood function, C - coincidence counting. Post-trial significance: not specified if less than 2.5 $\sigma$. Contribution to the flux: estimate of the fraction of the astrophysical flux assuming a power-law fit for tracks $\left(\nu_{\mu}\right)$ or cascades $\left(\nu_{e, \tau}\right)$ associated with the sources of a given sample. 
the liklyhood function, nor reconstructed energies of individual neutrinos (only muon energies $E_{\mu}$, see Sec. 1.2 and Fig. 1, are published). The authors of Ref. 78 constructed their own likelyhood function, simplified compared to the IceCube function used in 77, which took into account the neutrino energies. The sensitivity of such an analysis is low, so it is not surprising that it neither confirmed nor excluded the result of Ref. [77. The work to test the hypothesis of a connection of neutrinos with VLBI-bright blazars continues with the data from Northern hemisphere experiments. Preliminary results 91 of the ANTARES collaboration indicate the presence of correlation of arrival directions, compatible with that found from IceCube [77.

Correlations (significance $3.2 \sigma$ post trial) of the arrival directions of IceCube high-energy track events published as separate lists and alerts, with blazars selected on the basis of other criteria, have been found in Ref. [68. The sample of sources used there was not complete by any criterion, but was a combination of heterogeneous data based on observations in different bands. The only requirement was the registration of gamma rays from the blazar by some instrument. Forty-eight coincidences of directions of arrival neutrinos to the blazars were found, with an expectation of $\sim 32$ for random directions. It is interesting to note that 39 of these 48 blazars are present in the RFC catalog, i.e. that is, they have a VLBIcompact component, indicating the presence of parsecscale relativistic jets, and 16 of them belong to the complete sample used in Refs. [67, 77]. Therefore, the effect found in Ref. [68] is actually saturated by sources of the same class.

Thus, blazars, that is, active galaxies with relativistic jets pointing almost exactly toward the observer (but not necessarily bright in the gamma-ray band investigated by Fermi LAT), given the contribution of sources not listed in the catalogs, can explain the entire astrophysical neutrino flux, as estimated from the IceCube muon tracks, when extrapolated down to energies of the order of $\mathrm{TeV}$ by a power law (4). In the context of the two-component model discussed in Sec. 2.2, this corresponds to $100 \%$ of the hard flux component.

\subsubsection{Search for flares}

A blind search for neutrino flares from arbitrary directions in the sky opens up even more freedom in the parameters - moment and duration of the flare are added to the direction in the celestial sphere. The probability of finding a spatio-temporal cluster of events resulting from random fluctuations is very high, and studies of this kind in the presence of a large random background make sense only for predetermined sources, whose variability in the electromagnetic radiation could be associated with neutrino bursts. The most highly variable objects among potential neutrino sources are blazars and one-time catastrophic events on the stellar scales, see e.g,
Ref. 92. Neutrino flares are often searched in spatiotemporal coincidence with such events or with flares of electromagnetic radiation from blazars. Note that often the "neutrino flare" means the moment of arrival of a single rare (high-energy) event with a high probability of astrophysical origin. However, these results should be interpreted with caution: for the Poisson statistics, a single event may signal both an increase in the average expected rate of arrival of events (a flare), or a completely random single event at an expected rate of less than one event per observation time. The estimation of the neutrino flux in this case is ambiguous 93 .

a. AMANDA and $1 E S 1959+650$. Historically, the first claim of a coincidence of neutrino and gamma-ray flares of a blazar was made by the AMANDA collaboration based on 2002 data 94, 95. From the direction of the blazar 1ES $1959+650$, over 4 years of observations, 5 neutrino events were observed at the expectation of 3.7, which by itself is consistent with fluctuations. However, 3 of these 5 events came in a time interval of 66 days, coinciding with flares of this source in $\mathrm{TeV}$ gamma rays. The collaboration refrained from assessing the statistical significance of this coincidence because it was detected a posteriori, not in a "blind" analysis. Theoretical interpretations of this observation were controversial 96,97 . New similar gamma-ray flares of the same blazar in 2016 were not accompanied by neutrino excesses at IceCube; this, however, does not contradict the predictions of the proposed models 98 . It was this observation by AMANDA which motivated [4] the development of a system of mutual neutrino and gamma-ray telescope alerts, which has been actively developing already in the IceCube era.

b. IceCube and TXS 0506+056. One of the most widely known cases of a coincidence of a detected IceCube neutrino event with a gamma-ray blazar flare is the neutrino alert IC170922A, associated with the blazar TXS 0506+056. Unlike its AMANDA predecessors, the IceCube collaboration in this case, quite similar to the one described above, has evaluated and published [15] $a$ posteriori the statistical significance of this coincidence. In the description of this well-known event, we follow the original work [15.

A muon track, corresponding with a high probability to an astrophysical neutrino with energy of hundreds of $\mathrm{TeV}$ (an estimate of the energy of this event is shown in detail in Fig. 1), was recorded on 22 September 2017. The standard analysis demonstrated a coincidence of the arrival direction with the position of the gamma-ray bright blazar TXS 0506+056, which was in a period of increased activity. In response to the IceCube alert, this source was observed by atmospheric Cherenkov telescopes recording gamma rays with energies $\gtrsim 100 \mathrm{TeV}$ : on September 23 - by HESS and VERITAS and on September 24 by MAGIC. Gamma rays from this source were not detected by the three instruments; however, when MAGIC repeated its observation on September 28, it detected a non-zero signal corresponding to the increase of the flux 
in comparison with the upper limits from September 2324. Multi-wavelength observations demonstrated that at the time of the neutrino arrival, the blazar was at the beginning of a prolonged flare in the radio band; its Xray flux was quite modest (this observation has severely limited theoretical models of the neutrino origin). In the optical band, a few minutes after the neutrino detection, a strange, short drop in the brightness of the source was observed 99.

Blasars are numerous and highly variable sources, so the probability to accidentally find a blazar in a flaring state in a given direction and at a given moment in time is quite high. In the absence of an a priori fixed procedure, a correct estimate of the probability of such a coincidence is difficult: the main problem is to choose, what to call "a flare" of the source and what to call "a coincidence" with the flare. The authors of Ref. 15] proposed and applied several methods for assessing the statistical significance, described in the Supplementary Information to their paper. For these estimates, only correlations with Fermi LAT were used, since only this instrument provides continuous observations of blazars across the entire sky, to which a particular detected flare could be compared. The probability of a random coincidence, calculated a posteriori and not accounting for different trials, was $2.1 \times 10^{-5}(4.1 \sigma$ pre trial $)$. The authors of the paper counted observations of other neutrino events (at the time of the event analysis, there were a total of 51 events selected using the same criteria as IC170922A), for which there was no overlap with gamma-ray-bright blazar outbursts, as independent trials. Taking into account the corresponding penalty factor results in the probability $\approx 10^{-3}(3.0 \sigma$ post trial). This estimate does not account for the additional penalty factor for testing four models of the gamma-flux correlation, three of which can be considered independent, and for the choice of the Fermi-LAT light curve simulation parameters (bin size in time and the choice of the energy bin). Note also that after the publication of this result, IceCube has changed the criteria for selecting alert events, so that a statistically correct analysis of how many other similar neutrino events were not accompanied by flares of gamma-ray sources since then is not possible.

From the facts that only for one gamma-ray source such a coincidence has been reported, and that population analyses do not indicate a significant association of gamma-ray bright blazars with neutrinos, see Sec. 2.3.3. it has been suggested that the blazar TXS $0506+056$ is a "special" source, so the history of detection of neutrinos of all energies from this direction was analysed. A flare of neutrino events (19 events with an expectation of 6 ) with energies of $(0.1-20) \mathrm{TeV}$ and a significance of $3.5 \sigma$ (post trial) was found [100] from that specific direction in the sky. The time period in which this neutrino flare happened, 2014, was not special in terms of the gamma-ray activity of the source [101]. Note that using the IceCube event reconstruction published in 2021 [19, 20, the pretrial probability of registering this flare as a result of a

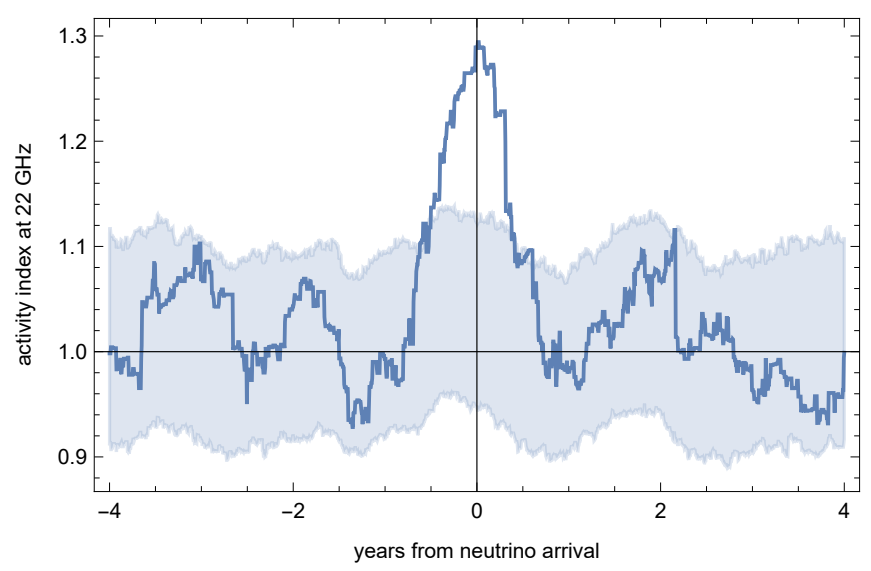

FIG. 17. Coincidence of the arrival moments of $E_{\nu}>$ $200 \mathrm{TeV}$ neutrinos with flares of blazars selected from the VLBI catalog (22 GHz RATAN-600 data from Ref. 67]). The activity index is defined as the ratio of the radio flux in the optimized period of 0.9 year to the flux for the entire period of observations. Averaged over 18 sources coinciding with the neutrino arrival directions. The shaded area is the expectation for random arrival directions (68\% CL).

random fluctuations is only $8.1 \times 10^{-3}$ [19, which is $\sim 100$ times higher than in the original analysis. With the same change in the post-trial significance it would amount to $\approx 2 \sigma$.

Numerous papers have investigated multiwavelength characteristics of this source in order to give a theoretical description of the neutrino production, see e.g., [102109. It was not possible to propose a model that simultaneously describes the events of 2014 and 2017 within a single mechanism. A study of the characteristics of the TXS $0506+056$ source demonstrated that it is a typical radio blazar [110]. Thus, the observations [15, 100] may not testify to the singularity of this source, but serve to illustrate the established population connection of neutrinos and radio blazars [67, 77]; the gamma-ray flare might be only a simultaneous signal of the source activity.

c. Flares of radio blazars. This interpretation motivated the search for coincidence of arrival moments of high-energy IceCube neutrinos with radio flares of the blazars 67] located in the same directions. The result of this study is illustrated in Fig. 17. from which one can see that, on average, the blazars coinciding with the neutrino arrival directions are in the period of the radio flare at the time of the neutrino arrival. Despite the impressive appearance of the figure, the post-trial statistical significance of this result is only slightly greater than $2 \sigma$ due to the penalty factor for the selection of the optimal time-window width. This result, obtained with the data of the blazar monitoring program on the RATAN600 telescope (Special Astrophysical Observatory of the Russian Academy of Sciences), was confirmed by observations of the American OVRO and Finnish Metsahovi observatories 90. 
d. Cosmic gamma-ray bursts. These stellar-scale processes with extreme energy release [111-113] have long been considered as potential sources of high-energy protons and neutrinos, see e.g. 114 117, but no space-time correlation between neutrinos and detected gamma-ray bursts has been found [118 121]. This narrows the range of gamma-ray bursts - potential sources of high-energy neutrinos - to optically thick ones not observed in electromagnetic radiation, for which such an analysis is not possible.

e. Tidal disruption of stars. These catastrophes occur when a star collides with a black hole and are observed primarily as flares in the optical band. Rarely, these events are accompanied by the formation of a jet. The absence of spatiotemporal correlations with the population of such sources limits their contribution to the IceCube astrophysical neutrino flux to the level of $\lesssim 1 \%$ (for processes with the jet formation, $\lesssim 26 \%$ ) [122]. There has been some interest in the IceCube neutrino event IC191001A with an energy of $\sim 217 \mathrm{TeV}$, which coincided in arrival direction with one of the tidal disruption events, AT2019dsg, although the neutrino came 154 days after it [123]. The authors of that paper give only an estimate of the probability of a random coincidence, $\approx 4.8 \times 10^{-3}$, without a detailed statistical analysis. This coincidence is discussed in more detail in Ref. 124. Note that in this case, the tidal disruption of the star was not accompanied by the formation of a relativistic jet.

f. Galactic sources. As discussed below in Sec. 3.1. for sources in our Galaxy, it is possible to observe highenergy neutrinos along with associated gamma rays of the same energy range. Gamma-ray astronomy above $100 \mathrm{TeV}$ is now being intensively developed, motivated in large part by the neutrino results of IceCube. The observations of point sources 125 130 and of diffuse gamma rays from the Galactic plane [131 in this band are worth mentioning. So far, the only indication of a possible connection of the flare of gamma rays with energies above $100 \mathrm{TeV}$ from a Galactic source and of neutrinos of the same energy comes from the Carpet-2 facility (Baksan Neutrino Observatory, INR RAS), which has recorded 132 the increased flux, coinciding with the arrival time of a IceCube neutrino with energy $\sim 154 \mathrm{TeV}$, from the region of the Galactic disk in the Cygnus constellation, which includes the so-called Cygnus Cocoon, a probable source of the most energetic cosmic photon ever recorded (1.4 PeV [130]). In Fig. 18, the dependence of the rate of arrival of atmospheric showers from the direction of this source is shown. The observed excess corresponds to a flare in photons with energies $\gtrsim 300 \mathrm{TeV}$ lasting about 3 months around the day of the neutrino event. The statistical significance of this flare is $3.1 \sigma$ (post trial). Note that although it is the Cygnus Cocoon that coincides with the the most likely direction of neutrino arrival, both the accuracy of this direction and the angular resolution of Carpet- 2 are worse than $4^{\circ}$, and other interesting sources are present in this uncertainty region, including relativistic binary systems detected in

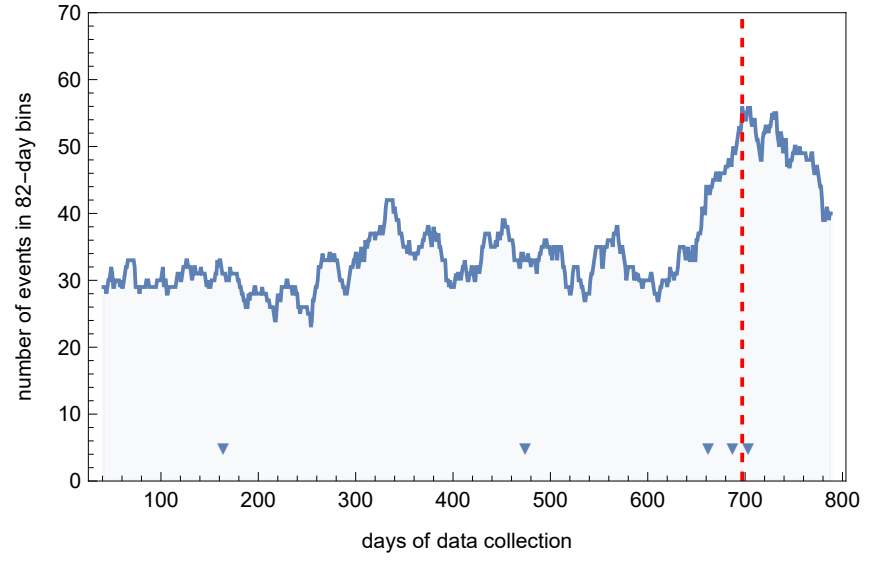

FIG. 18. Count rate of events with estimated primary energy above $300 \mathrm{TeV}$ from the direction of Cygnus Cocoon, as recorded by the Carpet- 2 facility. The vertical dashed line indicates the arrival time of a neutrino with energy $\sim 154 \mathrm{TeV}$ from this direction. The triangles at the bottom of the figure indicate the arrival times of the events that have been most rigorously filtered out as candidates for primary photons. Plotted with the data from Ref. 132.

gamma rays above $100 \mathrm{GeV}$.

\section{GENERAL CONSTRAINTS ON MODELS OF NEUTRINO ORIGIN}

\subsection{The $\pi$-meson mechanism and the multimessenger approach}

Neutrinos have no electric charge and therefore cannot be efficiently accelerated by external fields. Thus, highenergy neutrinos can only be produced in decays or interactions of other energetic or heavy particles. Examples of these processes are decays of ultrarelativistic nuclei (we will mention this mechanism in the end of this section), decays or annihilation of very heavy slow particles (dark matter, see Sec. 4.2). However, the most natural, guaranteed under astrophysical conditions mechanism is associated with the decays of $\pi$-mesons born in the interactions of cosmic rays with matter or radiation.

Indeed, experiments indicate the presence in our Galaxy (and, probably in other galaxies as well!) of highenergy hadrons - cosmic-ray particles, protons and nuclei [45, 133. These particles interact with the surrounding matter and radiation. At high energies, most of the interactions involving hadrons result in the production of the lightest of strongly interacting particles, $\pi$ mesons. The latter are unstable and their decay products include high-energy neutrinos. These processes must go wherever there are high-energy hadrons (cosmic rays), but their intensity depends on the amount of matter in the medium or of the target photons.

a. Proton-proton interactions. Without loss of generality, we will assume that both relativistic hadrons and 


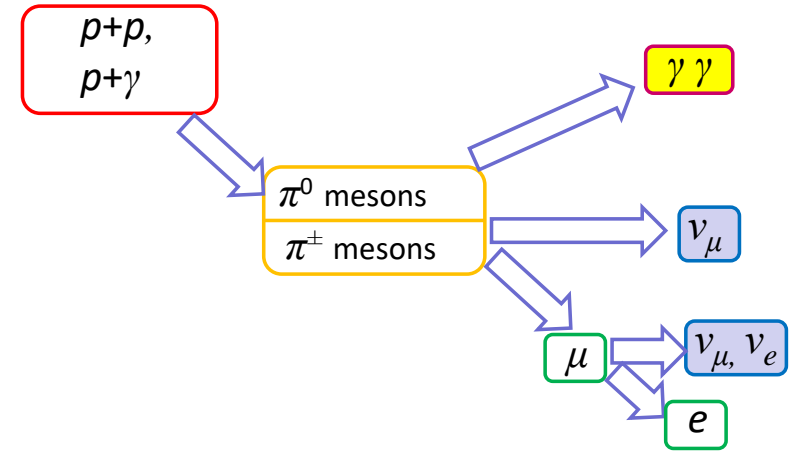

FIG. 19. Basic processes of neutrino production in astrophysical sources [14].

ambient hadrons are protons $(p)$. At high energies, $p p$ interactions proceed with the birth of one or more $\pi$ mesons [134, 135, with the leading $\pi$-meson carrying away on average $\sim 1 / 5$ part of the energy of the relativistic proton, $E_{p}$, in the rest frame of the target proton. The birth of $\pi^{0}, \pi^{+}$and $\pi^{-}$mesons are approximately equally probable, and high-energy emission is determined by decays (see Fig. 19)

$$
\begin{gathered}
\pi^{0} \rightarrow \gamma \gamma, \\
\pi^{+} \rightarrow \mu^{+} \nu_{\mu}, \\
\mu^{+} \rightarrow e^{+} \nu_{e} \bar{\nu}_{\mu}
\end{gathered}
$$

(the decay chain of $\pi^{-}$and $\mu^{-}$is obtained from Eqs. (6), (7) by charge conjugation). The energy of a charged $\pi$ meson is divided, on average about equally, among the four leptons in the final state, or more precisely [1, 136]

$$
\begin{gathered}
\frac{\left\langle E_{\nu_{\mu}}\right\rangle}{E_{\pi^{+}}}=\frac{1}{2}\left(1-\left(\frac{m_{\mu}}{m_{\pi}}\right)^{2}\right) \simeq 0.21 \\
\frac{\left\langle E_{\nu_{e}}\right\rangle}{E_{\pi^{+}}}=\frac{1}{10}\left(2+\left(\frac{m_{\mu}}{m_{\pi}}\right)^{2}\right) \simeq 0.26, \\
\frac{\left\langle E_{\bar{\nu}_{\mu}}\right\rangle}{E_{\pi^{+}}}=\frac{\left\langle E_{e_{+}}\right\rangle}{E_{\pi^{+}}}=\frac{1}{20}\left(3+4\left(\frac{m_{\mu}}{m_{\pi}}\right)^{2}\right) \simeq 0.27 .
\end{gathered}
$$

The energy of the neutral $\pi$-meson is divided equally between the two produced photons. Thus, as a result of the interaction of a proton with $E_{p}$ with a proton at rest, on average, either two photons with energies of $E_{p} / 10$ each, or (with the probability of $2 / 3$ ) three (anti)neutrinos with energies of $E_{p} / 20$ are produced. b. Proton-photon interactions. Consider now the interaction of relativistic protons with photons of the ambient radiation. At extremely high energies, this process also proceeds with multiple $\pi$-meson production, and in terms of the resulting neutrinos is not much different from the $p p$-interaction. However, we will be more interested in the other regime. Indeed, significant concentrations of photons in astrophysical sources are reached only at relatively low energies, so the main contribution to the production of astrophysical neutrinos comes from interactions with the single $\pi$-meson production. Unlike for $p p$, the production of $\pi$ mesons in $p \gamma$ interactions is a threshold process, and the total energy must be sufficient to produce at least a $\pi$ meson at rest. The main channel for this is the resonant birth and subsequent decay of the $\Delta^{+}$baryon,

$$
p+\gamma \rightarrow \Delta^{+} \rightarrow\left\{\begin{array}{l}
n+\pi^{+} \\
p+\pi^{0}
\end{array}\right.
$$

( $n$ denotes the neutron).

The first thing that draws attention is that in this process, no leading $\pi^{-}$is produced, so the equality of $\nu_{e}$ and $\bar{\nu}_{e}$ at birth is violated. However, for the sum of neutrinos and antineutrinos, the flavor content obtained in $p \gamma$-interactions does not differ from the $p p$ case.

Further, the kinematics of the two-particle $\Delta^{+}$decay allows us to determine the fraction of the energy of the initial particles carried away by the $\pi$-meson: it is again $\sim 1 / 5$ (the numerical agreement with the $p p$ case is occasional and takes place only in the $\Delta$-resonance approximation), so, like for the $p p$-process, $E_{\nu} \sim E_{p} / 20$ and $E_{\gamma} \sim E_{p} / 10$ (but, due to the absence of $\pi^{-}$, photons are now born in about $1 / 2$ of cases rather than $1 / 3$ ).

Finally, the resonance condition will be written as $E_{p} E_{\gamma_{B}}=m_{\Delta}^{2}$, where $E_{\gamma_{B}}$ is the energy of the initial photon, and $m_{\Delta} \approx 1.23 \mathrm{GeV}$ is the $\Delta$ baryon mass. Therefore, for the production of a neutrino with energy $E_{\nu}$ in the $p \gamma$ process, we can estimate the required energy not only of the proton, $E_{p} \simeq 20 E_{\nu}$, but also of the initial photon,

$$
E_{\gamma_{B}}=\frac{m_{\Delta}^{2}}{20 E_{\nu}} \simeq 750 \mathrm{eV}\left(\frac{E_{\nu}}{100 \mathrm{TeV}}\right)^{-1}
$$

For practical applications, it is important to remember that Eq. $(9)$ is written in the source frame, so $E_{\nu}$ here may be different from the observed one, e.g., if the source is located at a cosmological redshift $z$ or if the source is moving relativistically along the line of sight. Note also that the estimates given here and in the description of the $p p$-process are easy to memorize but approximate and in a number of cases do not work, see, for example, Ref. [137.

In many models of acceleration of cosmic-ray particles, the spectrum of relativistic protons turns out to be power-like. For the scattering on protons at rest, the scale invariance of the $p p$-process leads to the fact 
that the spectra of secondary particles, and, eventually, neutrinos, repeat the power-law spectrum of the original protons. The resonant nature of the $p \gamma$-scattering, in contrast, leads to neutrino spectra that are different from the power law and are determined by the spectra of target photons.

c. Oscillations and flavor content. So, from the decays of $\pi^{ \pm}$-mesons, one obtains $\nu_{e}, \nu_{\mu}$ and corresponding antineutrinos. In $p p$-interactions, neutrinos and antineutrinos are born equally, in the $p \gamma$ case the production of $\bar{\nu}_{e}$ is suppressed in the $\Delta$-resonance approximation. In either case, the number of produced $\nu_{\mu}$ and $\bar{\nu}_{\mu}$ is twice that of $\nu_{e}$ and $\bar{\nu}_{e}$. However, on the way from the source to the observer, the flavor composition of the neutrinos changes due to oscillations.

Denote the difference in the squared masses of the two neutrino states as $\Delta m^{2}$, the neutrino energy as $E_{\nu}$. Then the oscillation length is

$L_{\mathrm{osc}}=4 \pi \frac{E_{\nu}}{\Delta m^{2}} \approx 22.4$ a.u. $\left(\frac{E_{\nu}}{100 \mathrm{TeV}}\right)\left(\frac{\Delta m^{2}}{7.4 \times 10^{-5} \mathrm{eV}^{2}}\right)^{-1}$

Given that the radius of the orbit of Uranus is about 19 a.u., we obtain that the distance to any neutrino source outside the Solar system is much larger than the oscillation length (to estimate this, the smallest $\Delta m^{2}$ in the system of three neutrino flavors was substituted into Eq. (10)). In addition, for most astrophysical sources their size is also noticeably larger than $L_{\text {osc }}$. Therefore, for the flavor composition of neutrinos at detection, it makes sense to consider only the average over the oscillation phases, which means that the so-called GribovPontecorvo approximation works. Within this approximation, the fractions of neutrinos of flavors $l=e, \mu, \tau$ at the observation point, $f_{l}$, are related to those at the source, $f_{l}^{0}$, by a simple relation,

$$
f_{l}=\sum_{l^{\prime}} M_{l l^{\prime}} f_{l^{\prime}}^{0}
$$

where the transformation matrix $M_{l l^{\prime}}=\sum_{i}\left|U_{l i}^{2}\right|\left|U_{l^{\prime} i}^{2}\right|$, and $U_{l i}$ is the neutrino mixing matrix (the PontecorvoMaki-Nakagawa-Sakata matrix, see e.g. Ref. [138]). The values of elements of this matrix, the neutrino oscillation parameters, are known with certain accuracy and continue to be refined in new experiments, see Ref. 139. For the purposes of this review, an approximation in which $f_{\tau}^{0}=0$ is sufficient, then $f_{\mu}^{0}=1-f_{e}^{0}$ and

$$
\left\{\begin{array}{l}
f_{e} \simeq 0.18+0.36 f_{e}^{0} \\
f_{\mu} \simeq 0.44-0.25 f_{e}^{0} \\
f_{\tau} \simeq 0.38-0.11 f_{e}^{0}
\end{array}\right.
$$

Notably, for the value $f_{e}^{0} \approx 1 / 3$ expected from $\pi$-meson decays in the source, we obtain an approximate equality of the detection probabilities of the three neutrino flavors at the detection point ${ }^{2}$.

d. The multimessenger approach. Since low-energy photons and target protons are abundant in many astrophysical objects, the mechanism described above links fluxes of three types of high-energy particles, the astrophysical messengers, - neutrinos from $\pi^{ \pm}$decays, photons from decays of $\pi^{0}$ and the original relativistic protons, cosmic rays. This link underlies the so-called multimessenger approach to observational constraints on source models. Constraints of this type have been used to estimate high energy astrophysical neutrino fluxes for decades before their discovery [140 142, and after the discovery they became a powerful quantitative tool to understand the neutrino origin.

We have seen that in $\pi$-meson decays, the emitted flux of neutrinos of energies $E_{\nu}$ is accompanied by an emitted flux of photons at energies $E_{\gamma} \sim 2 E_{\nu}$. The energy fluxes (measured, for example, in $\mathrm{TeV} / \mathrm{cm}^{2} / \mathrm{s}$ ) carried by photons $\gamma$ and neutrinos $\nu$, are related as

$$
E_{\gamma}^{2} \frac{d N_{\gamma}}{d E_{\gamma}}=\left.A E_{\nu}^{2} \frac{d N_{\nu}}{d E_{\nu}}\right|_{E_{\nu}=E_{\gamma} / 2},
$$

where $A \sim 2 / 3(4 / 3)$ for the $p p(p \gamma)$ mechanism, respectively. However, unlike neutrinos, high-energy photons do not propagate through the Universe freely: they produce electron-positron pairs $e^{+} e^{-}$in interactions with photons of the background radiation [143. This process is a threshold process, and its cross section peaks strongly just above the threshold, when the energies of the initial photons satisfy the ratio $E_{\gamma} E_{\gamma_{B}} \approx 4 m_{e}^{2}$, where $m_{e}$ is the electron mass. It is convenient to rewrite this relation in the form

$$
\frac{E_{\gamma}}{\mathrm{TeV}} \cdot \frac{E_{\gamma_{B}}}{\mathrm{eV}} \approx 1
$$

from where it follows that photons with $E_{\gamma} \sim(100-$ 1000) $\mathrm{TeV}$ produce pairs mostly on cosmic microwave background photons, whose density in the Universe is very high. As a consequence, the mean free path of the photons born jointly with neutrinos does not exceed the size of the Galaxy, or its immediate vicinity. This, however, is only a part of the story, since the produced $e^{ \pm}$ interact with the same background radiation, transferring their energy to photons (inverse Compton scattering). The resulting photons, each with energy already slightly lower than the energy of the initial one, again produce electron-positron pairs, and the electromagnetic cascade continues (Fig. 20). After each cycle, the energy of the original photon is redistributed among secondary photons, so that the average energy of the photons in

2 The expression 11 is valid for both neutrinos and antineutrinos. Given that, with the exception of the Glashow resonance, it is impossible to distinguish $\nu$ and $\bar{\nu}$ by modern experiments, we do not distinguish between them in these expressions. 


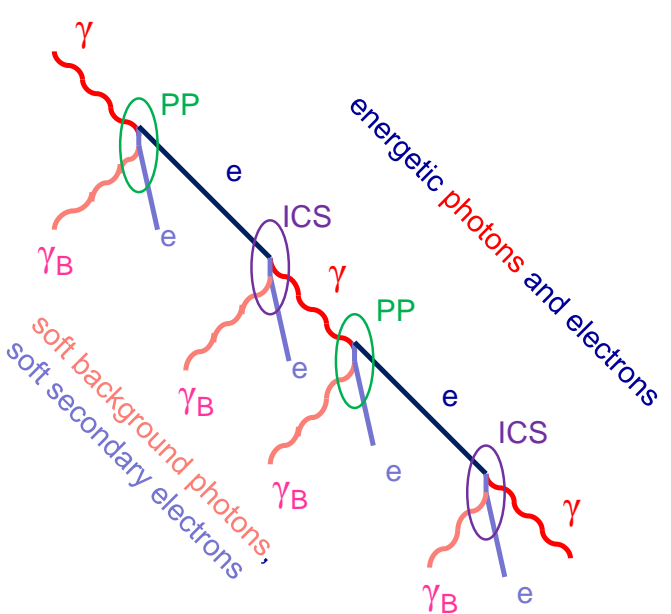

FIG. 20. The electromagnetic cascade development [14. PP - pair production, ICS - inverse Compton scattering.

the cascade decreases, although the total energy is conserved (see Refs. [141, 144]). This continues until the average energy of the photons drops so much that they continue moving through the Universe unimpeded. This occurs at photon energies of several tens of $\mathrm{GeV}$, so that all energy of the extragalactic diffuse gamma rays with $E_{\gamma} \gtrsim 100 \mathrm{TeV}$ is "pumped" into the $\sim 10 \mathrm{GeV}$ range. The flux of isotropic gamma rays in this range have been measured [145] by the LAT instrument aboard the Fermi satellite, so that the flux obtained by cascading companion photons from neutrino sources in no way can exceed these measurements. Such a requirement allows one to set an upper limit on the diffuse neutrino flux from extragalactic sources [140] unless they themselves absorb all photons with energies $\gtrsim 10 \mathrm{GeV}$. The quantitative value of this limit depends, although not too much, on assumptions about the shape of the neutrino spectrum, on the mechanism ( $p p$ or $p \gamma$ ) and, most importantly, on the distribution of sources in the Universe (evolution). On the other hand, the contribution of Galactic sources can be estimated from observations of photons with $E_{\gamma} \sim 2 E_{\nu}$, which within the Galaxy can reach the observer almost without absorption, see Fig. 21. In the context of IceCube measurements, these possibilities have been investigated, for example, in the papers [148, 157-161].

A similar, though requiring much more assumptions, construction can be built also for cosmic rays. This widely known estimate has been called the WaxmanBahcall limit [142, although, as we shall see below, it gives some characteristic value of the flux rather than constrains it from above. Suppose that all cosmic rays of ultra-high energy detected by appropriate detectors are accelerated in some extragalactic sources, and determine the number of high-energy protons from measured fluxes at energies $\sim 10^{19} \mathrm{eV}$ (at these energies cosmic rays are guaranteed to be extragalactic in origin). The same mechanism that accelerates some protons to the highest energies would accelerate many more protons to

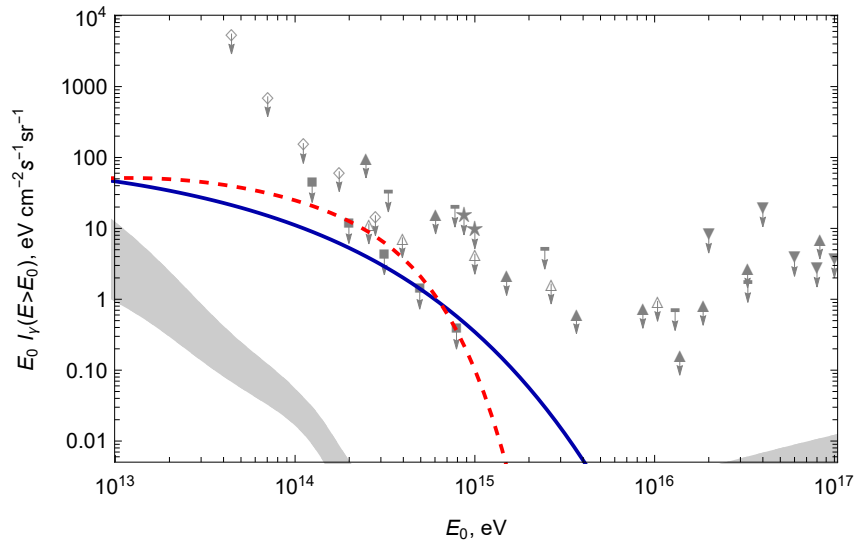

FIG. 21. Predictions of the integral isotropic diffuse flux of high-energy photons in the models explaining the observed neutrino flux: Galactic (solid line - interaction of cosmic rays with gas in the walls of the Local Bubble [146, dashed linedark-matter particle decays [147]) and extragalactic (shaded region [148). The gray symbols are experimental upper limits (empty rhombuses - GRAPES-3 [149, 150, empty triangles - KASCADE 151, upward triangles - KASCADE and KASCADE-Grande [152, downward triangles - EASMSU [153, horizontal dashes - CASA-MIA [154], asterisks - EAS-TOP [155], squares - preliminary analysis of Tibet$\mathrm{AS} \gamma$ in Ref. [156]).

lower energies, $\sim\left(10^{16}-10^{17}\right) \mathrm{eV}$. In contrast to the most energetic ones, the protons at lower energies are trapped by the magnetic field in the source, and sooner or later interact there with matter or radiation, giving rise to $\pi$ mesons. The neutrino flux that would be produced if the energy of all these protons were transferred to the $\pi$ mesons, decaying into photons and neutrinos 142, gives by construction an upper limit on neutrino fluxes (this energy may remain in cosmic rays or be reemitted in other ways). However, soon after the publication of this estimate, it became clear that it uses too many assumptions [162, first of all, the rather finely tuned assumption that cosmic rays with energies $\sim 10^{19} \mathrm{eV}$ leave the source, but protons of lower energy, those producing the observed neutrinos, stay there. It is unclear whether cosmic rays of ultra-high energies and neutrinos of energies $\sim 100 \mathrm{TeV}$ must be produced in the same sources, since to accelerate protons to $\sim 10^{19} \mathrm{eV}$, much more exotic, difficult to achieve conditions are required than those necessary for the neutrino-producing protons of $E_{p} \sim 20 E_{\nu} \sim 10^{16} \mathrm{eV}$. Finally, there is still no clarity, at which energies cosmic rays detected at the Earth have the extragalactic origin, while the numerical value of the obtained estimate depends considerably on this.

The appealing simplicity of these estimates has been one of the important motivations for building cubickilometer scale neutrino telescopes, whose sensitivity just reaches such flux values [163. Surprisingly, the observed IceCube neutrino fluxes are, by the order of magnitude, close to these estimates, understood as upper limits, although originally this could not have been predicted: 
"Experiments with extragalactic neutrinos..., given a positive result, will provide very important and unique information both for astrophysics and for elementary-particle physics. If the result is negative (with an installation of $10^{9} \mathrm{~m}^{3}$ ), this will merely give cause to the mournful arguments by the astrophysicists, who will undoubtedly find many "natural" explanations for the low flux of extragalactic neutrinos" (V.S. Berezinsky, G.T. Zatsepin [163]).

One might even say that one of the major difficulties in theoretical explanation of the origin of the detected astrophysical neutrinos is precisely that their flux turned out to be very large, at the level of maximal expectations. And these estimates have remained in the focus also of more detailed studies of the neutrino origin.

Let us take a look at Fig. 22. Similar plots are often used to illustrate the multimessenger approach. It shows the energy fluxes of diffuse photons, neutrinos, and cosmic rays recorded by different instruments in the corresponding energy ranges. One can note that the fluxes of neutrinos $\sim \mathrm{PeV}$, photons $\sim 10 \mathrm{GeV}$, and protons $\sim 10^{19} \mathrm{eV}$ are of the same order of magnitude, $\sim 10^{-8} \mathrm{GeV} / \mathrm{cm}^{2} / \mathrm{sec}$. This allows one to speculate about the realization of the scenario described above, saturating the multimessenger constraints (neutrinos are born in extragalactic sources of ultra-high energy cosmic rays, and the diffuse gamma rays are the result of electromagnetic cascades triggered by photons born jointly with the neutrinos). For neutrinos with energies above $100 \mathrm{TeV}$ such models can indeed 166 be proposed. However, on closer examination, for the neutrino flux of all energies, such reasoning looks superficial and not necessarily correct:

1. the neutrino fluxes registered by IceCube in the cascade mode, at energies below $\sim 50 \mathrm{TeV}$, violate the Fermi-LAT upper limit and exceed the WaxmanBahcall estimate;

2. a significant fraction of the diffuse photon flux recorded by Fermi LAT is associated with numerous distant blazars, which are not resolvable into individual point sources due to imperfections of the instrument [167; and these blazars may be neutrino sources themselves;

3. the cosmic-ray spectrum decreases rapidly, and the assertions of approximate equality of energy fluxes are only valid for certain energies; it is unclear how these energies are singled out compared to the somewhat smaller, $\sim 3 \times 10^{17} \mathrm{eV}$, at which cosmic rays are also likely extragalactic (for quantitative estimates, see Ref. [168]).

As discussed above, other observational evidence also suggests that the picture of the neutrino origin is more complex. e. Other regimes and mechanisms. Let us briefly mention possible variations of the standard picture based on the $\pi$-meson decays, discussed above, while remaining within the Standard Model of particle physics.

The muon-damp regime. If the decays of $\pi^{ \pm}$-mesons occur in the region of a strong magnetic field, the relatively long-lived $\mu^{ \pm}$have time to lose a significant fraction of their energy to synchrotron radiation before decaying. In this case, all of the high-energy neutrinos are muon neutrinos directly born from $\pi^{ \pm}$, that is, in the formulas 12 we should put $f_{e}^{0}=0$. Oscillations result in a flavor composition different from the standard one: the average flux of $\nu_{e}$ and $\nu_{\tau}$ is now not equal to that of $\nu_{\mu}$, but to $\sim 2 / 3$ of it. The current accuracy of the determination of composition is still insufficient to reliably distinguish between these scenarios, see Fig. 12. This, and even more exotic, scenarios are discussed, for example, in Refs. 169, 170. The magnetic field, at which this mode starts to work, can be estimated from the requirement that the characteristic synchrotron-loss time of the muon is less than its lifetime, while the muon energy can be related to that of the neutrino. It turns out that to damp the muons, the field must exceed

$$
B_{\mu \mathrm{damp}} \approx 60 \mathrm{kG}\left(\frac{E_{\nu}}{100 \mathrm{TeV}}\right)^{-1}
$$

Fields of order $10^{4} \mathrm{G}$ may be present in the immediate neighborhoods of supermassive black holes, so further refinement of the flavor composition of observed neutrinos could isolate or constrain this class of potential sources 171. Note that the number of photons from $\pi^{0}$ decays per high-energy neutrino in this case is larger than for the usual scenario.

Note about beta decays. The presence of a significant number of nuclei heavier than protons among cosmic-ray particles, in principle, allows for the following scenario for the origin of neutrinos. A nucleus, like a proton, is accelerated to significant energy and then decays, for example, by photodesintegration. Some of its fragments, primarily individual neutrons, may be unstable with respect to beta decay. Neutrinos will be present among the decay products and inherit part of the kinetic energy of the original nucleus. This part, however, is small, since the fraction of the neutrino energy relative to the energy of the neutron is, in the laboratory frame, only $\sim 10^{-3}$. Therefore, as a rule, the contribution of such a mechanism to the production of high-energy neutrinos is small compared to that of conventional hadronic interactions. The threshold for photodesintegration of nuclei is about an order of magnitude below the threshold for the $\pi$-meson production, so there is a short energy range in which this process is significant 172. Muon neutrinos are not produced in this case, $f_{e}^{0}=1$, and the observed composition, according to Eq. 12, is also depleted in $\nu_{\mu}$, which is in a poor agreement with the IceCube results, even taking into account their low precision [24, 39]. 


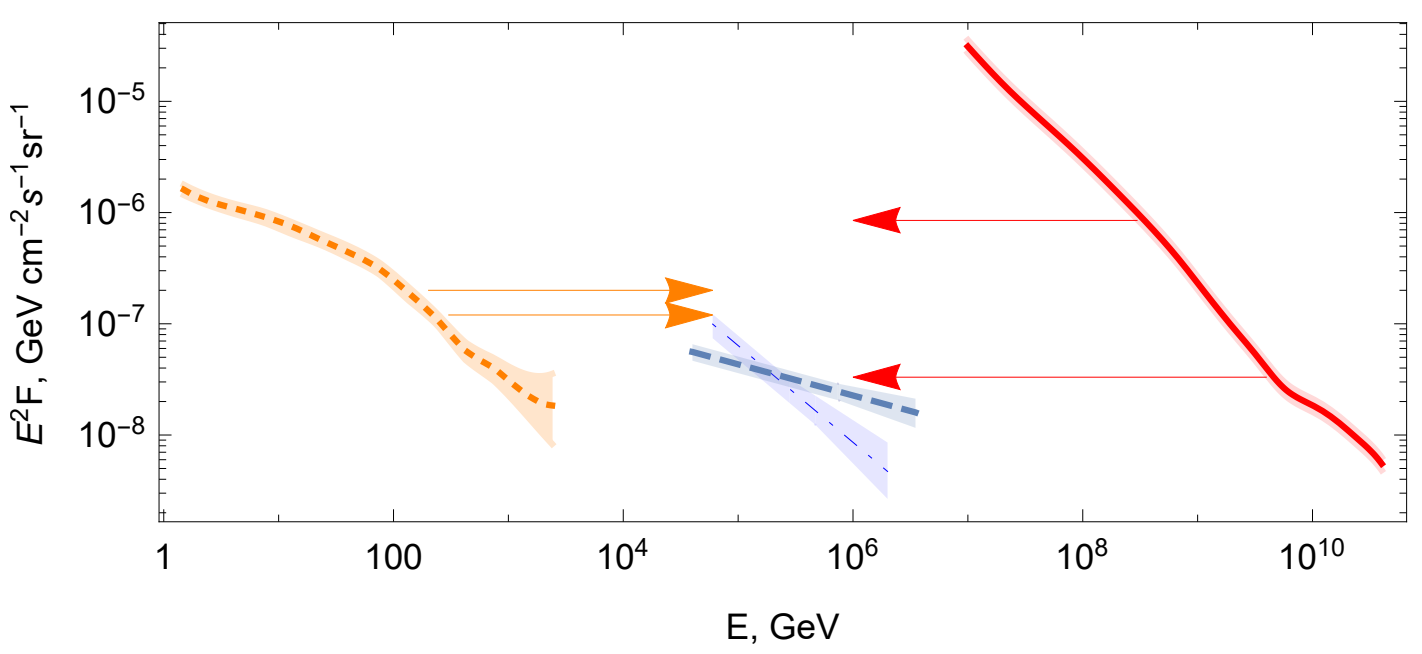

FIG. 22. Constraints on and estimates of the neutrino flux in the multimessenger approach. The orange dashed line on the left, $E \sim(1-1000) \mathrm{GeV},-$ diffuse isotropic gamma ray flux according to Fermi LAT data [164]. In the middle, $E \sim\left(10^{5}-10^{6}\right) \mathrm{GeV}$, - total fluxes of astrophysical neutrinos of all flavors according to IceCube data (blue dashed line - $\nu_{\mu} 2019$ [25]; thin blue dot-dashed line - HESE 2020 24] $)$. On the right, $E \sim\left(10^{7}-10^{10}\right) \mathrm{GeV},-$ cosmic-ray flux according to the combined fit [165]. The arrows illustrate the estimates and constraints discussed in the text and their uncertainties.

\subsection{General constraints on source populations}

Each particular model of the neutrino origin gives its own predictions of the distribution of arrival directions in the sky and can be tested either in a combination of specific individual analyses or on the basis of the results outlined in Sec. 2.3. However, there exist approaches in which, starting with observational data, it is possible to obtain general constraints on the number and the neutrino luminosity of the sources from the main population contributing to the neutrino flux. In the simplest form, mostly used today, the sources within the population are assumed to be similar; one must, however, keep in mind that realistic assumptions about natural diversity, e.g., of the luminosities of sources within the same class, may significantly alter the results of such an analysis (for a similar problem for cosmic rays of ultrahigh energies, see Refs. [173 175]).

a. Estimate of the number of sources and their neutrino luminosity. It has already been noted above that the absence of statistically significant individual sources in the neutrino sky indicates that the observed flux either has a diffuse origin, or is produced by a large number of astrophysical objects, each contributing a little to the observed neutrino flux. Here we give quantitative estimates of these parameters.

Assume that the observed flux is produced by sources with the same neutrino luminosity and some redshift distribution (so called cosmological evolution of sources). One can then estimate how often, for a given experimental exposure, the observation of more than one (two, three, ...) neutrinos from the source ("multiplets") is expected. Comparing the number of multiplets in the data with that expected for a random distribution allows one to constrain the density of the number of sources in the Universe for a fixed evolution. In a situation where the constraint is due to the lack of observation of significant clusters of events corresponding to point sources, it is easy to show that the assumption of the same luminosity leads to the most conservative constraint. The method of constraining the number of sources based on the statistics of arrival-direction clustering has been proposed in Ref. 176] for ultra-high energy cosmic rays, but its application to neutrino astronomy is considerably different, see e.g., Ref. [177. The main difference is that cosmic rays, because of interactions with the background radiation, are collected from a limited volume of the Universe, and the absence of clusters means a large number of sources in that volume, that is, a large local source density. The neutrinos are collected from all over the Universe, and the contribution of (in any case numerous) distant sources dominates. The lack of clusters for neutrinos indicates that there are no nearby sources standing out against the uniform background of distant and weak ones, i.e., the large distance to the nearest source or a small local density ${ }^{3}$ Of course, in the analysis one have to account for the large number of random clusters from atmospheric neutrinos. On the other hand, unlike charged cosmic rays, neutrinos propagate in straight lines, so the angular size of the cluster is completely determined by the precision of the reconstruction of arrival directions.

Knowing the average neutrino luminosity of each source and the distribution of sources in distances, i.e.,

3 A lack of clusters both in cosmic rays and in neutrinos therefore suggests that it would be unlike to observe cross-correlations between their arrival directions [178. 


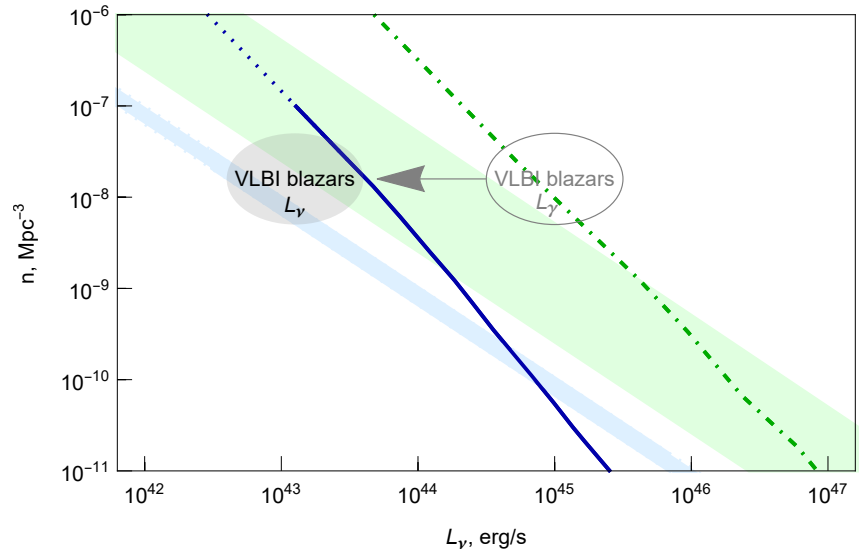

FIG. 23. Constraints on the source number density $n$ (at redshift $z=0$ ) and their typical neutrino luminosity $L_{\nu}$. The dashed line - upper limit (99\% CL) on $n$ from the absence of significant neutrino point sources in the track sample of 8 years of IceCube data 185; wide shaded band - allowed $(99 \%$ CL) region in which these sources explain the flux and spectrum of IceCube from the same data 185. The solid line (the dotted line is its extrapolation) is the upper limit ( $95 \%$ CL) on $n$ from the absence of clusters of neutrino arrival directions in the sample of IceCube tracks with energies $>200 \mathrm{TeV}$ 183; the narrow shaded band is the allowed $(68 \%$ CL) region in which these sources explain the flux and spectrum from the same IceCube data 183. The constraints assume a strong positive evolution typical for active galactic nuclei. The shaded ellipse is an estimate of the characteristic parameters of VLBI-selected blazars, see Ref. 2.3.3. The empty ellipse is the same, but assuming equality of neutrino and bolometric gamma-ray luminosities.

the input data for estimating the statistics of event clusters, it is easy to calculate the total neutrino flux and to compare it with observations. The combination of the two requirements, the absence of significant point sources or clusters of events and explanation of the observed flux, allows one to constrain the combinations of neutrino luminosity and source number density for a fixed assumption about their cosmological evolution. Variations of this approach have been applied to constraining the parameters of neutrino sources in Refs. [179 185. In Fig. 23. results are presented for models with fast positive evolution (many sources at large redshifts), characteristic for active galactic nuclei. The constraints for high $\left(E_{\nu}>200 \mathrm{TeV}\right)$ and all (i.e., mostly $\left.\gtrsim 10 \mathrm{TeV}\right)$ energies, obtained from track events, are shown separately. The combination of the required neutrino luminosity and the statistics of arrival-direction clusters places serious constraints on the models of sources. In particular, the neutrino luminosity of a typical source appears to be substantially smaller than the characteristic photon luminosity of objects of this type. The most severe constraints for specific classes of sources arise if, in addition to clustering, i.e., autocorrelation of neutrino arrival directions, one takes into account the cross-correlation of neutrinos and directions to the sources, see Sec. 2.3.3 184. For instance, in Fig. 23 the region of the characteristic parameters of VLBI-selected blazars, Sec. 2.3.3 [67, 77], is shown. To explain the observed flux, their neutrino luminosity must be of order of a few per cent of the luminosity in photons. It can be seen that in this case, they can be sources of all astrophysical neutrinos, both of those with energies above $200 \mathrm{TeV}$, and with lower energies, in agreement with the observed directional correlations.

As for the sources with weak evolution, constraints similar to those shown in Fig. 23 are satisfied only for low neutrino luminosity and high source number densities. For densities of $\gtrsim 10^{-6} / \mathrm{Mpc}^{3}$ these constraints require to take into account 186 the local large-scale structure of the Universe, which the distributions of all astrophysical objects follow, when analyzing neutrino arrival directions; examples of sources of this type are starburst galaxies. The analyses carried out [187, 188, have not yielded strong constraints on these scenarios yet.

\subsection{Conclusions about general constraints}

Multimessenger analysis of diffuse fluxes of extragalactic cosmic rays, GeV gamma rays, and high-energy astrophysical neutrinos demonstrates that the neutrino fluxes detected by IceCube are at the level of upper limits, i.e., the highest theoretical expectations, for extragalactic sources with the $\pi$-meson mechanism. Of course, particular quantitative limits depend on the details of the model, but qualitatively the picture indicates that the observed particle fluxes in the three channels - cosmic rays, neutrinos and photons, - are consistent with each other by the order of magnitude. This allows for the possibility that proton interactions play a significant role in the origin not only of neutrinos, but also of high-energy gamma rays. Note that prior to the IceCube observations, the origin of the bulk of the astrophysical high-energy gamma rays was usually attributed to relativistic electrons.

At the same time, results of the studies of neutrino arrival directions, primarily their diffuse nature - the lack of clearly identified sources providing for a significant contribution to the flux; the lack of neutrino correlations with populations of gamma-ray bright potential sources; strong constraints on the Galactic-disk excess in the neutrino flux, - point to difficulties of the naive multimessenger approach. It is not possible to satisfy all constraints simultaneously and to explain all observations in a simple model with a single class of sources. It is possible that the observed flux of high-energy astrophysical neutrinos comes from different classes of sources, which include both galactic "PeVatrons" which dominate at lower energies, and extragalactic ones (e.g, radio blazars), which provide a harder spectrum. At the same time, the number of extragalactic sources contributing to the observed flux is large, and the neutrino luminosity of each of them is orders of magnitude smaller than the photon luminosity. 


\section{POTENTIAL SOURCE CLASSES}

Staying within the frameworks of the general picture derived from observational constraints and described at the end of the previous section, we very briefly list here specific astrophysical objects and environments which are potential sources of observed neutrinos.

\subsection{Models of extragalactic sources}

a. Active galactic nuclei. Environments of supermassive black holes in active galactic nuclei are the most powerful steady sources of radiation in the Universe. Relativistic blazar jets directed towards the observer result in the strong enhancement of fluxes of both photons and neutrinos due to the Lorentzian kinematics. They have been considered as sources of high-energy neutrinos in theoretical works since the early days of neutrino astronomy 189 191, see also recent reviews 192 195] and references therein.

The classical scenario $196-202$ involves the neutrino production in the vicinity of a black hole, closer than the jet launches. The density of matter in the central regions of galactic nuclei is low, and the main channel for the neutrino production is probably the $p \gamma$ process. Relativistic protons with the required energies up to $\sim\left(10^{16}-10^{17}\right) \mathrm{eV}$ can be accelerated either in the black-hole magnetosphere 203] or in shock waves in the vicinity of the accretion disk. The latter is also the source of intense radiation that provides the second necessary ingredient, the target photons.

In the context of the observational data discussed above, this scenario requires extension or modification because it does not allow one to explain the astrophysical neutrinos of energies $\sim 10 \mathrm{TeV}$, for which a connection to blazars has also been established [77. Indeed, according to Eq. (9), production of these neutrinos requires X-ray target photons, while the accretion disk emits mainly in the ultraviolet and can provide the target photons for the production of neutrinos of much higher energies only. Modifications of this scenario include a contribution from the emission of the accretion disk corona 204 207 whose spectrum, compared to the disk itself, extends into higher energies, although with a lower intensity. A common problem of models of the neutrino production in the region of the disk and its corona is the lack of a direct link to relativistic amplification in the jets - as a rule, neutrinos in such models are emitted isotropically. An additional, albeit avoidable, complication is related to the fact that, judging by the fast variability, the observed gamma rays from the blazars originate precisely in this region, while no statistically significant association between neutrinos and such gamma rays were found.

These problems are circumvented if neutrinos are born already in the relativistic jets, near their base, see e.g., [77, 208, 209]. Here, X-ray photons of the target are present, and the acceleration of protons to the desired en- ergies can occur in weakly relativistic shock waves $210-$ 212 . This is where the visible radio emission of blazars is produced, correlations of neutrino arrival moments with enhancements in which have been established in 67, 90 (regions closer to the black hole are opaque to radio emission). Since one-zone models fail to describe the entire spectrum of the observed electromagnetic radiation of blazars from the radio to gamma rays, it is natural to assume that the neutrino radiation may not come from the same region as the gamma radiation. However, it is worth noting that the target photon density in the jet is small, and to produce the required number of neutrinos in this region one needs a significant proton power of the jet [77. It could be possible that the observed correlation between neutrinos and the radio emission indicates the operation of the $p p$-mechanism [213].

b. Cosmic-ray reservoirs. This class includes astrophysical objects of various scales where cosmic rays are trapped for long periods by magnetic field. The probability of their interaction with ambient protons grows with time, so that eventual neutrino production by the $p p$-mechanism is guaranteed. One of the most widely discussed source classes of this kind are starburst galaxies, which have magnetic fields large enough to confine protons and where mechanisms to accelerate cosmic protons to high energies may work. Ref. 214, prior to the start of IceCube data taking, predicted diffuse neutrino flux from a population of starburst galaxies, at the level of the subsequently discovered flux. Sources of this class are prototypical for the Waxman-Bacall estimate, and all the multimessenger relations and constraints discussed in Sec. 3.1 work for them. In particular, such sources are transparent to gamma rays, but are not blazars, so the "cascade" limit from Fermi LAT is particularly serious [215, 216]. The reservoirs of cosmic rays, which in addition to starburst galaxies include also clusters of galaxies 217, 218, and kiloparsec-scale structures in active galaxies, radio lobes 219, can therefore be sources of observable neutrinos only with energies $\gtrsim 100 \mathrm{TeV}$ [220]. The interest in this class of sources is heated [221] by an excess of IceCube events from the direction of a nearby powerful starburst galaxy NGC 1068, see Sec. 2.3.2, but no analysis of the source population confirms a significant contribution from cosmic-ray reservoirs to the neutrino flux.

c. Stopped jets. In explosions of very massive stars, not uncommon in particular at the early stages of the galaxies' evolution, the jets produced in the central part of the star may not reach the surface because of the high density of the hydrogen envelope of the star. The result is a "choked gamma-ray burst," an event with the energy release of a cosmic gamma-ray burst but without a detectable flare of electromagnetic radiation, which is absorbed by the outer layers of the star and/or the surrounding matter. In the process of interaction of the jets with matter, neutrinos are born in $p p$ interactions $[222$ 225., and because of the lack of visible gamma-ray bursts, there is no contradiction with the constraints from the 
population analysis 226, 227. Theoretical models of such sources contain considerable uncertainties, but a common feature is a strong peak in the energy distribution of neutrinos near $100 \mathrm{TeV}$. As a consequence, such sources cannot explain the entire observed neutrino flux - either the observed flux at $\sim 100 \mathrm{TeV}$ is explained, but not below or above, or the predicted flux at $100 \mathrm{TeV}$ is too high 228233 .

d. Tidal disruption of stars. Falling of a star into a supermassive black hole is a rare event. On average, in a particular galaxy it happens once per every $10^{4}-10^{5}$ years. The star is first destroyed by tidal forces and then about half of its matter accretes onto the black hole. A sharp increase in the accretion rate leads in any case to a flare and, rarely, to the formation of a jet. One can understand this event as a short-term transformation of an ordinary galaxy into an active one, so that neutrinos can be produced in the jet in the usual $p \gamma$ process. Moreover, the remnants of the star provide additional target material for the $p p$ process. The majority of theoretical works on the neutrino production in tidal disruption events address processes with the formation of jets $234-240$, to which the case of a registered coincidence with the IceCube event 123 does not seem to belong [124, 241]. The contribution of tidal disruption events without jet formation to the observed highenergy neutrino flux can only be very small, as follows both from a theoretical point of view [241] and from population analysis 122 .

\subsection{Models of the Galactic flux component}

When discussing possible scenarios for the origin of neutrinos in our Galaxy, one should not forget about strict observational constraints on neutrinos from the Galactic disk, see Sec. 2.3.1, Fig. 15. The constraints on the disk contribution (and the first indications of its observations) are at the order of $10 \%$ of the total neutrino flux, in agreement with expectations for the guaranteed flux from cosmic-ray interactions with the interstellar gas. Successful scenarios for the origin of the dominant part of the neutrino flux in the Galaxy must somehow circumvent these disk-related constraints. There are two possibilities:

1. the flux comes from sources not related to the disk, i.e. distributed in the Galactic halo (circumgalactic gas or halo dark matter);

2. the main contribution to the flux comes from the immediate neighborhood of the Solar system, that is, from the region smaller in size than the thickness of the disk.

In addition, the sources can be individual rare objects or regions in the Galactic plane whose distribution does not follow the distribution of the disk's gamma rays, which is assumed in the derivation of constraints of Sec. 2.3.1. a. Circumgalactic gas. In recent years, a variety of observational evidence has been obtained (242]; see e.g. Ref. 243 and references therein) for the existence of an extended halo of circumgalactic gas around our Galaxy, extending up to its virial radius, i.e. about $200 \mathrm{kpc}$ (recall that the radius of the Galactic disk is about $20 \mathrm{kpc}$, and its thickness is $<1 \mathrm{kpc}$ ). At energies of $\gtrsim 10^{17} \mathrm{eV}$, cosmic protons leave the Galactic disk and, interacting with the gas, can produce high-energy photons 244 and neutrinos 245. Under the assumption of symmetric diffusion of cosmic rays and for a realistic model of the circumgalactic gas, the neutrino flux from such interactions is only a few percent of the observed one 246, but it may be possible that either feeding the halo with cosmic rays through Fermi bubbles, or other manifestations of past activity in our Galactic nucleus, could alter this result [247].

b. Decays of dark-matter particles. Convincingly confirmed by astronomical observations at various scales, the existence of invisible matter in the Universe has yet to be firmly explained in terms of particle physics. There are many working models, attracting more and more attention while the scenario of weakly interacting massive particles, most popular for decades, is gradually being excluded experimentally, see, e.g., the discussion in Ref. 248. Astrophysical neutrinos can be produced in the decays of dark-matter particles $249 \sqrt[252]{2}$; to produce decay products of the energies discussed, the dark-matter particles must be superheavy [253, 254]. In the context of explaining the IceCube results, this scenario has been discussed in particular in Refs. 255 260. The entire observed neutrino spectrum from tens of $\mathrm{TeV}$ to tens of $\mathrm{PeV}$ cannot be explained in this way, because the energy distribution of the decay products is noticeably narrower, but this mechanism can explain the observed flux at some energies. Even for purely lepton decay channels, very strict constraints on such a scenario are imposed from the lack of observation of an accompanying flux of high-energy photons [261 264.

c. Gas bubbles and star formation regions. In the Galactic disk, neutrinos can be produced in interactions of cosmic rays with protons and nuclei of the interstellar gas, but both are unevenly distributed across the disk. Cosmic rays are accelerated in sources, "PeVatrons," and are trapped in regions of high magnetic field, while the distribution of gas is complex because of stellar winds and shock waves from supernova explosions. As a consequence, the neutrino signal from the disk may be dominated by the contribution of a few regions of intense star formation [265 268], which look like compact clusters of young massive stars, and so-called superbubbles. A special place is the Local Bubble, within which our Solar System resides: the neutrinos coming from it [146, 147, 269] do not point back to the Galactic disk since the size of the bubble, $\sim 100 \mathrm{pc}$, is noticeably smaller than the thickness of the disk. Sources of this type include the Cygnus Cocoon, mentioned in Sec. 2.3.4 in connection with registration from of a flare of photons with energies above $300 \mathrm{TeV}$, coinciding with the IceCube neutrino 
event 132 . Note that the available constraints will also be satisfied by neutrinos from other rare objects, whose distribution in the sky does not follow the profile of the Galactic gas, such as microquasars in gamma-ray bright binary systems, one of which coincides, within the accuracy of determining the arrival direction of neutrinos and photons discussed above, with the Cygnus Cocoon [270].

d. Contribution of similar galaxies. Speaking of the Galactic origin of neutrinos, we should not forget that our Galaxy is not unique and sources like these are present not only in it, but also in billions of other galaxies. Although the contribution of the sources in our immediate vicinity is significant, neutrinos are collected from all over the Universe, and the total contribution from other galaxies may be of the same order as ours. Simple quantitative estimates [271] show that this is indeed the fact: by the order of magnitude, any Galactic contribution to the persistent flux of high-energy neutrinos would be similar to the total contribution of similar sources in all other galaxies in the Universe.

\section{CONCLUSIONS}

- Astrophysical neutrinos of high, $10 \mathrm{TeV}-10 \mathrm{PeV}$, energies have been reliably detected by the IceCube experiment; their observation is being confirmed by the ANTARES results and by the first Baikal-GVD data. The distribution in zenith angles of events passing the most strict selection excludes their atmospheric origin even for exotic assumptions. In the energy range above $100 \mathrm{TeV}$, neutrino astronomy has surpassed photon astronomy and motivated its development.

- Although the sources of high-energy astrophysical neutrinos have not yet been definitively determined, their total fluxes point to a significant role of relativistic hadrons in high-energy astrophysics: within standard physics, only processes involving them can give rise to neutrinos of this energy range.

- Spectra of astrophysical neutrinos reconstructed from IceCube cascade and track events are poorly consistent with each other under the assumption of a power-law dependence of the flux on the energy. This can be explained by a more complex shape of the spectrum, reflecting the combined contribution of different populations of sources. It is likely that the sky in neutrinos looks no less complex and diverse as in photons.

- The flux of astrophysical neutrinos at energies above $\sim 100 \mathrm{TeV}$ is probably dominated by the contribution of numerous distant extragalactic sources. Population studies indicate a statistically significant association of neutrinos with blazars, that is the active galactic nuclei with relativistic jets directed toward the observer, manifesting themselves by powerful radiation from parsec scales visible with VLBI. These sources are not always bright in the gamma-ray band, and their neutrino luminosity on average is noticeably lower than the photon luminosity. They contribute significantly to the neutrino flux also at lower energies.

- The neutrino flux component that dominates at energies $\sim(10-100) \mathrm{TeV}$ may either be of a Galactic origin or be connected to multiple extragalactic sources which are opaque to photons at $\gtrsim \mathrm{GeV}$ energies. There are observational indications to the presence of a Galactic component.

- The prospects for further understanding of the nature of high-energy astrophysical neutrinos are related both to the work of cubic-kilometer scale neutrino telescopes (including Northern-hemisphere water detectors, Baikal-GVD, which has just started to work, and KM3NeT which is under construction) and to the multimessenger analysis, including observations in the electromagnetic channel in all bands - from radio (in which, due to the best angular resolution, it is possible to study blazar jets in the immediate vicinity of supermassive black holes) to $\mathrm{PeV}$ (this emerging field of astronomy will answer questions about Galactic sources of neutrinos).

The author is indebted for interesting and helpful discussions of various aspects related to the origin of high-energy astrophysical neutrinos to his colleagues, M. Barkov, A. Bykov, H. Dembinski, T. Dzhatdoev, Zh.-A. Dzhilkibaev, G. Domogatsky, A. Franckowiak, O. Kalashev, Yu.A. and Yu.Yu. Kovalev, M. Kuznetsov, T. Montaruli, K. Murase, A. Neronov, A. Plavin, E. Podlesny, K. Postnov, V. Rubakov, G. Rubtsov, K. Ryabtsev, D. Samtleben, D. Semikoz, K. Spiering, O. Suvorova and K. Zhuravleva.

This work is supported by the Ministry of Science and Higher Education of the Russian Federation, Contract $075-15-2020-778$ of the Program of Major Scientific Projects within the National Project "Science".
[1] A. Capone, P. Lipari, and F. Vissani, In: Multiple Messengers and Challenges in Astroparticle Physics (2018).

[2] M. Spurio, Probes of Multimessenger Astrophysics:
Charged cosmic rays, neutrinos, $\gamma$-rays and gravitational waves (Springer, 2018).

[3] A. Palladino, M. Spurio, and F. Vissani, Universe 6, 30 
(2020), arXiv:2009.01919 [astro-ph.HE]

[4] C. Spiering, Phys. Usp. 57, 470 (2014), arXiv:1402.2096 [astro-ph.IM]

[5] C. Spiering, Neutrino Detectors Under Water and Ice. In: Fabjan C., Schopper H. (eds) Particle Physics Reference Library. Springer, Cham. (2020).

[6] V. A. Ryabov, Phys. Usp. 49, 905 (2006)

[7] S. L. Glashow, Phys. Rev. 118, 316 (1960)

[8] V. S. Berezinsky and A. Z. Gazizov, JETP Lett. 25, 254 (1977).

[9] M. G. Aartsen et al. (IceCube), Astrophys. J. 835, 151 (2017), arXiv:1609.04981 [astro-ph.HE]

[10] J. G. Learned and S. Pakvasa, Astropart. Phys. 3, 267 (1995), arXiv:hep-ph/9405296

[11] M. G. Aartsen et al. (IceCube), Phys. Rev. D 93, 022001 (2016), arXiv:1509.06212 [astro-ph.HE]

12] M. G. Aartsen et al. (IceCube), Nature 591, 220 (2021).

[13] R. Abbasi et al. (IceCube), (2020), arXiv:2011.03561 [hep-ex]

[14] S. Troitsky (2019) in "Multimessenger astronomy", A. Cherepaschuk (Ed.), Vek-2, Fryazino.

[15] M. G. Aartsen et al. (IceCube, Fermi-LAT, MAGIC, AGILE, ASAS-SN, HAWC, H.E.S.S., INTEGRAL, Kanata, Kiso, Kapteyn, Liverpool Telescope, Subaru, Swift NuSTAR, VERITAS, VLA/17B-403), Science 361, eaat1378 (2018), arXiv:1807.08816 [astroph.HE]

[16] T. Chiarusi and M. Spurio, Eur. Phys. J. C 65, 649 (2010), arXiv:0906.2634 [astro-ph.HE]

[17] M. G. Aartsen et al. (IceCube), Astrophys. J. 833, 3 (2016), arXiv:1607.08006 [astro-ph.HE]

[18] IceCube Collaboration 10.21234/B4KS6S (2018, doi:10.21234/B4KS6S).

[19] IceCube Collaboration (IceCube) (2021, doi:10.21234/sxvs-mt83).

[20] R. Abbasi et al. (IceCube) 10.21234/CPKQ-K003 (2021), arXiv:2101.09836 [astro-ph.HE]

[21] M. G. Aartsen et al. (IceCube), Phys. Rev. Lett. 113, 101101 (2014), arXiv:1405.5303 [astro-ph.HE]

[22] M. G. Aartsen et al. (IceCube), in 34th International Cosmic Ray Conference (2015) arXiv:1510.05223 [astroph.HE]

[23] M. G. Aartsen et al. (IceCube), , 54 (2017), arXiv:1710.01179 [astro-ph.HE]

[24] R. Abbasi et al. (IceCube), Phys. Rev. D 104, 022002 (2021), arXiv:2011.03545 [astro-ph.HE]

[25] J. Stettner (IceCube), PoS ICRC2019, 1017 (2020), arXiv:1908.09551 [astro-ph.HE]

[26] M. A. Markov, in 10th International Conference on High Energy Physics (1960) p. 578.

[27] I. Zheleznykh, Int. J. Mod. Phys. A 21S1, 1 (2006)

[28] L. A. Fusco and F. Versari (ANTARES), PoS ICRC2019, 891 (2020)

[29] A. D. Avrorin et al. (Baikal-GVD), PoS ICRC2019, 873 (2021) arXiv:1908.05430 [astro-ph.HE]

30] M. G. Aartsen et al. (IceCube), Phys. Rev. Lett. 125, 121104 (2020), arXiv:2001.09520 [astro-ph.HE].

[31] M. G. Aartsen et al. (IceCube), Phys. Rev. D 91, 022001 (2015), arXiv:1410.1749 [astro-ph.HE]

[32] M. G. Aartsen et al. (IceCube), Phys. Rev. D 99, 032004 (2019), arXiv:1808.07629 [hep-ex]

[33] D. Fargion, P. Oliva, and G. Ucci, PoS FRAPWS2014, 028 (2016), arXiv:1512.08794 [astro-ph.HE].

[34] C. Mascaretti and F. Vissani, JCAP 08, 004,
arXiv:1904.11938 [astro-ph.HE]

[35] D. Fargion, P. G. De Sanctis Lucentini, M. Y. Khlopov, P. Oliva, F. La Monaca, and P. Paggi, PoS FRAPWS2018, 007 (2019), arXiv:1911.07240 [astroph.HE]

[36] A. Palladino, C. Mascaretti, and F. Vissani, JCAP 08, 004 arXiv:1804.04965 [astro-ph.HE]

[37] C.-Y. Chen, P. S. Bhupal Dev, and A. Soni, Phys. Rev. D 92, 073001 (2015), arXiv:1411.5658 [hep-ph]

[38] F. Halzen, PoS ICRC2019, 021 (2020) arXiv:1909.09468 [astro-ph.HE]

[39] M. Bustamante and M. Ahlers, Phys. Rev. Lett. 122, 241101 (2019), arXiv:1901.10087 [astro-ph.HE]

[40] N. Song, S. W. Li, C. A. Argüelles, M. Bustamante, and A. C. Vincent, JCAP 04, 054, arXiv:2012.12893 [hep$\mathrm{ph}]$.

[41] A. C. Vincent, S. Palomares-Ruiz, and O. Mena, Phys. Rev. D 94, 023009 (2016), arXiv:1605.01556 astroph.HE]

[42] A. Palladino and F. Vissani, Astrophys. J. 826, 185 (2016), arXiv:1601.06678 [astro-ph.HE]

[43] A. Neronov and D. Semikoz, Phys. Rev. D 93, 123002 (2016), arXiv:1603.06733 [astro-ph.HE]

[44] A. Palladino, M. Spurio, and F. Vissani, JCAP 12, 045. arXiv:1610.07015 [astro-ph.HE],

[45] S. V. Troitsky, Phys. Usp. 56, 304 (2013), arXiv:1301.2118 [astro-ph.HE]

[46] M. G. Aartsen et al. (IceCube), Astrophys. J. 835, 151 (2017), arXiv:1609.04981 [astro-ph.HE]

[47] IceCube Collaboration (IceCube)

$(2019$ doi:10.21234/exm3-tm26).

[48] M. G. Aartsen et al. (IceCube), , 31 (2017), arXiv:1710.01179 [astro-ph.HE],

[49] S. Ando, I. Tamborra, and F. Zandanel, Phys. Rev. Lett. 115, 221101 (2015), arXiv:1509.02444 [astro-ph.HE].

[50] D. Gaggero, D. Grasso, A. Marinelli, A. Urbano, and M. Valli, Astrophys. J. Lett. 815, L25 (2015), arXiv:1504.00227 [astro-ph.HE]

[51] M. G. Aartsen et al. (IceCube), Astrophys. J. 886, 12 (2019), arXiv:1907.06714 [astro-ph.HE]

[52] A. Albert et al. (ANTARES, IceCube), Astrophys. J. Lett. 868, L20 (2018), arXiv:1808.03531 [astro-ph.HE]

[53] A. Neronov and D. V. Semikoz, Astropart. Phys. 75, 60 (2016), arXiv:1509.03522 [astro-ph.HE]

[54] S. Troitsky, JETP Lett. 102, 785 (2015), arXiv:1511.01708 [astro-ph.HE]

[55] S. L. Dubovsky and P. G. Tinyakov, JETP Lett. 68, 107 (1998), arXiv:hep-ph/9802382

[56] C. A. Argüelles and H. Dujmovic (IceCube), PoS ICRC2019, 839 (2020), arXiv:1907.11193 [hep-ph].

[57] A. Dekker, M. Chianese, and S. Ando, JCAP 09, 007, arXiv:1910.12917 [hep-ph].

[58] M. G. Aartsen et al. (IceCube), Eur. Phys. J. C 78, 831 (2018), arXiv:1804.03848 [astro-ph.HE]

[59] A. Bhattacharya, A. Esmaili, S. Palomares-Ruiz, and I. Sarcevic, JCAP 05, 051, arXiv:1903.12623 [hep-ph].

[60] M. Su, T. R. Slatyer, and D. P. Finkbeiner, Astrophys. J. 724, 1044 (2010), arXiv:1005.5480 [astro-ph.HE]

[61] S. Hallmann, T. Eberl, et al. (ANTARES), PoS ICRC2017, 1001 (2018)

[62] C. Lunardini, S. Razzaque, K. T. Theodoseau, and L. Yang, Phys. Rev. D 90, 023016 (2014), arXiv:1311.7188 [astro-ph.HE],

[63] K. Fang, M. Su, T. Linden, and K. Murase, Phys. Rev. 
D 96, 123007 (2017), arXiv:1704.03869 [astro-ph.HE]

[64] P. Tinyakov and I. Tkachev, Phys. Rev. D 69, 128301 (2004), arXiv:astro-ph/0301336

[65] M. G. Aartsen et al. (IceCube), Phys. Rev. Lett. 124, 051103 (2020), arXiv:1910.08488 [astro-ph.HE].

[66] A. Albert et al. (ANTARES, IceCube), Astrophys. J. 892, 92 (2020), arXiv:2001.04412 [astro-ph.HE]

[67] A. Plavin, Y. Y. Kovalev, Y. A. Kovalev, and S. Troitsky, Astrophys. J. 894, 101 (2020), arXiv:2001.00930 [astro-ph.HE]

[68] P. Giommi, T. Glauch, P. Padovani, E. Resconi, A. Turcati, and Y. L. Chang, Mon. Not. Roy. Astron. Soc. 497, 865 (2020), arXiv:2001.09355 [astro-ph.HE]

[69] M. G. Aartsen et al. (IceCube), Astrophys. J. 898, 117 (2020), arXiv:2003.12071 [astro-ph.HE]

[70] D. Smith, D. Hooper, and A. Vieregg, JCAP 03, 031 arXiv:2007.12706 [astro-ph.HE]

[71] A. Albert et al. (ANTARES), Astrophys. J. 911, 48 (2021), arXiv:2012.15082 [astro-ph.HE]

72] P. Padovani, A. Turcati, and E. Resconi, Mon. Not. Roy. Astron. Soc. 477, 3469 (2018) arXiv:1804.01386 astroph.HE]

[73] A. Neronov, D. V. Semikoz, and K. Ptitsyna, Astron. Astrophys. 603, A135 (2017) arXiv:1611.06338 [astroph.HE]

[74] A. Palladino and F. Vissani, Astron. Astrophys. 604, A18 (2017), arXiv:1702.08779 [astro-ph.HE]

[75] P. Padovani, E. Resconi, P. Giommi, B. Arsioli, and Y. L. Chang, Mon. Not. Roy. Astron. Soc. 457, 3582 (2016), arXiv:1601.06550 [astro-ph.HE]

[76] M. Huber (IceCube), PoS ICRC2019, 916 (2020), arXiv:1908.08458 [astro-ph.HE]

[77] A. V. Plavin, Y. Y. Kovalev, Y. A. Kovalev, and S. V. Troitsky, Astrophys. J. 908, 157 (2021), arXiv:2009.08914 [astro-ph.HE].

[78] B. Zhou, M. Kamionkowski, and Y.-f. Liang, Phys. Rev. D 103, 123018 (2021), arXiv:2103.12813 [astro-ph.HE]

[79] F. Acero et al. (Fermi-LAT), Astrophys. J. Suppl. 218, 23 (2015), arXiv:1501.02003 [astro-ph.HE]

[80] M. Ackermann et al. (Fermi-LAT), Astrophys. J. Suppl. 222, 5 (2016), arXiv:1508.04449 [astro-ph.HE]

[81] M. Ajello et al. (Fermi-LAT), Astrophys. J. Suppl. 232, 18 (2017), arXiv:1702.00664 [astro-ph.HE],

82] M. Ackermann et al. (Fermi-LAT), Astrophys. J. 810, 14 (2015), arXiv:1501.06054 [astro-ph.HE].

83 M. Ajello et al. (Fermi-LAT), Astrophys. J. 892, 105 (2020), arXiv:1905.10771 [astro-ph.HE]

[84] Y.-L. Chang, C. Brandt, and P. Giommi, Astron. Comput. 30, 100350 (2020), arXiv:1909.11455 [astro-ph.HE]

[85] Y. L. Chang, B. Arsioli, P. Giommi, and P. Padovani, Astron. Astrophys. 598, A17 (2017), arXiv:1609.05808 [astro-ph.HE]

[86] Y.-L. Chang, B. Arsioli, P. Giommi, P. Padovani, and C. Brandt, Astron. Astrophys. 632, A77 (2019), arXiv:1909.08279 [astro-ph.HE]

[87] J. A. Zensus, Ann. Rev. Astron. Astrophys. 35, 607 (1997)

[88] R. Blandford, D. Meier, and A. Readhead, Ann. Rev. Astron. Astrophys. 57, 467 (2019), arXiv:1812.06025 [astro-ph.HE]

[89] A. R. Thompson, J. M. Moran, and J. Swenson, George W., Interferometry and Synthesis in Radio Astronomy, 3rd Edition (2017).

[90] T. Hovatta et al., Astron. Astrophys. 650, A83 (2021),
arXiv:2009.10523 [astro-ph.HE]

[91] J. Aublin and A. Plavin (ANTARES), PoS ICRC2021, 1164 (2021)

[92] K. Murase and I. Bartos, Ann. Rev. Nucl. Part. Sci. 69, 477 (2019), arXiv:1907.12506 [astro-ph.HE]

[93] N. L. Strotjohann, M. Kowalski, and A. Franckowiak, Astron. Astrophys. 622, L9 (2019), arXiv:1809.06865 [astro-ph.HE]

[94] M. Ackermann, Searches for signals from cosmic pointlike sources of high energy neutrinos in 5 years of AMANDA-II data (Humboldt Univ., Berlin , 2006) Ph. D. Thesis, available at https://edoc.huberlin.de/handle/18452/16219.

[95] E. Bernardini (IceCube), Nucl. Instrum. Meth. A 567, $418(2006)$

[96] F. Halzen and D. Hooper, Astropart. Phys. 23, 537 (2005), arXiv:astro-ph/0502449

[97] A. Reimer, M. Bottcher, and S. Postnikov, Astrophys. J. 630, 186 (2005), arXiv:astro-ph/0505233.

[98] T. Kintscher, K. Krings, D. Dorner, W. Bhattacharyya, and M. Takahashi (IceCube, FACT, MAGIC), PoS ICRC2017, 969 (2018).

[99] V. M. Lipunov et al., Astrophys. J. Lett. 896, L19 (2020), arXiv:2006.04918 [astro-ph.HE]

[100] M. G. Aartsen et al. (IceCube), Science 361, 147 (2018), arXiv:1807.08794 [astro-ph.HE]

[101] F. Halzen, A. Kheirandish, T. Weisgarber, and S. P. Wakely, Astrophys. J. Lett. 874, L9 (2019), arXiv:1811.07439 [astro-ph.HE]

[102] S. Gao, A. Fedynitch, W. Winter, and M. Pohl, Nature Astron. 3, 88 (2019), arXiv:1807.04275 [astro-ph.HE],

[103] M. Cerruti, A. Zech, C. Boisson, G. Emery, S. Inoue, and J. P. Lenain, Mon. Not. Roy. Astron. Soc. 483, L12 (2019), [Erratum: Mon.Not.Roy.Astron.Soc. 502, L21L22 (2021)], arXiv:1807.04335 [astro-ph.HE].

[104] A. Keivani et al., Astrophys. J. 864, 84 (2018) arXiv:1807.04537 [astro-ph.HE]

[105] K. Murase, F. Oikonomou, and M. Petropoulou, Astrophys. J. 865, 124 (2018), arXiv:1807.04748 [astroph.HE]

[106] A. Gokus, S. Richter, F. Spanier, M. Kreter, M. Kadler, K. Mannheim, and J. Wilms, Astron. Nachr. 339, 331 (2018), arXiv:1808.05540 [astro-ph.HE]

[107] N. Sahakyan, Astrophys. J. 866, 109 (2018), arXiv:1808.05651 [astro-ph.HE].

[108] A. Reimer, M. Boettcher, and S. Buson, Astrophys. J. 881, 46 (2019), [Erratum: Astrophys.J. 899, 168 (2020)], arXiv:1812.05654 [astro-ph.HE]

[109] X. Rodrigues, S. Gao, A. Fedynitch, A. Palladino, and W. Winter, Astrophys. J. Lett. 874, L29 (2019) arXiv:1812.05939 [astro-ph.HE]

[110] Y. A. Kovalev et al., Adv. Space Res. 65, 745 (2020).

[111] K. A. Postnov, Physics Uspekhi 42, 469 (1999).

[112] A. M. Bykov, Phys. Usp. 61, 805 (2018)

[113] R. L. Aptekar et al., Phys. Usp. 62, 739 (2019)

[114] M. Milgrom and V. Usov, Astrophys. J. Lett. 449, L37 (1995), arXiv:astro-ph/9505009.

[115] E. Waxman, Phys. Rev. Lett. 75, 386 (1995), arXiv:astro-ph/9505082

[116] M. Vietri, Astrophys. J. 453, 883 (1995), arXiv:astro$\mathrm{ph} / 9506081$

[117] E. Waxman and J. N. Bahcall, Phys. Rev. Lett. 78, 2292 (1997), arXiv:astro-ph/9701231

[118] M. G. Aartsen et al. (IceCube), Astrophys. J. 824, 115 
(2016), arXiv:1601.06484 [astro-ph.HE]

[119] M. G. Aartsen et al. (IceCube), Astrophys. J. 843, 112 (2017), arXiv:1702.06868 [astro-ph.HE]

[120] A. Albert et al. (ANTARES), Mon. Not. Roy. Astron. Soc. 500, 5614 (2020), arXiv:2008.02127 [astro-ph.HE]

[121] A. Albert et al. (ANTARES), JCAP 03, 092. arXiv:2011.11411 [astro-ph.HE]

[122] R. Stein (IceCube), PoS ICRC2019, 1016 (2020), arXiv:1908.08547 [astro-ph.HE]

[123] R. Stein et al., Nature Astron. 5, 510 (2021), arXiv:2005.05340 [astro-ph.HE].

[124] W. Winter and C. Lunardini, Nature Astron. 5, 472 (2021), arXiv:2005.06097 [astro-ph.HE]

[125] M. Amenomori et al., Phys. Rev. Lett. 123, 051101 (2019), arXiv:1906.05521 [astro-ph.HE]

[126] A. U. Abeysekara et al. (HAWC), Astrophys. J. 881, 134 (2019), arXiv:1905.12518 [astro-ph.HE].

[127] A. Albert et al., Astrophys. J. Lett. 907, L30 (2021), arXiv:2012.15275 [astro-ph.HE]

[128] A. U. Abeysekara et al. (HAWC), Phys. Rev. Lett. 124, 021102 (2020), arXiv:1909.08609 [astro-ph.HE],

[129] A. Albert et al. (HAWC), Astrophys. J. Lett. 896, L29 (2020), arXiv:2005.13699 [astro-ph.HE]

[130] Z. Cao et al. (LHAASO), Nature 594, 33 (2021).

[131] M. Amenomori et al., Phys. Rev. Lett. 126, 141101 (2021)

[132] D. D. Dzhappuev et al. (Carpet-3 Group), Astrophys. J. Lett. 916, L22 (2021), arXiv:2105.07242 [astro-ph.HE]

[133] M. Kachelriess and D. V. Semikoz, Prog. Part. Nucl. Phys. 109, 103710 (2019), arXiv:1904.08160 [astroph.HE]

[134] V. S. Berezinsky and V. V. Volynsky, in International Cosmic Ray Conference, International Cosmic Ray Conference, Vol. 10 (1979) p. 326.

[135] S. R. Kelner, F. A. Aharonian, and V. V. Bugayov, Phys. Rev. D 74, 034018 (2006), [Erratum: Phys.Rev.D 79, 039901 (2009)], arXiv:astro-ph/0606058.

[136] P. Lipari, M. Lusignoli, and D. Meloni, Phys. Rev. D 75, 123005 (2007), arXiv:0704.0718 [astro-ph].

[137] E. Roulet and F. Vissani, JCAP 03, 050 , arXiv:2011.12769 [astro-ph.HE]

[138] Y. G. Kudenko, Phys. Usp. 61, 739 (2018).

[139] P. A. Zyla et al. (Particle Data Group), PTEP 2020, 083C01 (2020)

[140] V. S. Berezinsky and A. Y. Smirnov, Astrophys. Space Sci. 32, 461 (1975)

[141] V. S. Berezinskii, S. V. Bulanov, V. L. Ginzburg, V. A. Dogel, and V. S. Ptuskin, Astrophysics of cosmic rays. (1984).

[142] E. Waxman and J. N. Bahcall, Phys. Rev. D 59, 023002 (1999), arXiv:hep-ph/9807282

[143] A. I. Nikishov, JETP 14, 393 (1962).

[144] V. Berezinsky and O. Kalashev, Phys. Rev. D 94, 023007 (2016), arXiv:1603.03989 [astro-ph.HE].

[145] M. Ackermann et al. (Fermi-LAT), Astrophys. J. 799, 86 (2015), arXiv:1410.3696 [astro-ph.HE]

[146] M. Bouyahiaoui, M. Kachelrieß, and D. V. Semikoz, Phys. Rev. D 101, 123023 (2020), arXiv:2001.00768 [astro-ph.HE]

[147] A. Neronov, M. Kachelrieß, and D. V. Semikoz, Phys. Rev. D 98, 023004 (2018), arXiv:1802.09983 [astroph.HE]

[148] O. E. Kalashev and S. V. Troitsky, Pisma Zh. Eksp. Teor. Fiz. 100, 865 (2014), arXiv:1410.2600 [astro-
ph.HE]

[149] M. Minamino et al. (GRAPES-3), in 31th International Cosmic Ray Conference (2009) p. 1089.

[150] H. Tanaka et al., J. Phys. G 39, 025201 (2012).

[151] G. Schatz et al., in 28th International Cosmic Ray Conference (2003).

[152] W. D. Apel et al. (KASCADE Grande), Astrophys. J. 848, 1 (2017), arXiv:1710.02889 [astro-ph.HE]

[153] Y. A. Fomin, N. N. Kalmykov, I. S. Karpikov, G. V. Kulikov, M. Y. Kuznetsov, G. I. Rubtsov, V. P. Sulakov, and S. V. Troitsky, Phys. Rev. D 95, 123011 (2017), arXiv:1702.08024 [astro-ph.HE]

[154] M. C. Chantell et al. (CASA-MIA), Phys. Rev. Lett. 79, 1805 (1997), arXiv:astro-ph/9705246

[155] M. Aglietta et al. (EAS-TOP), Astropart. Phys. 6, 71 (1996).

[156] A. Neronov, D. Semikoz, and I. Vovk, Astron. Astrophys. 653, L4 (2021), arXiv:2107.06541 [astro-ph.HE]

[157] K. Murase, M. Ahlers, and B. C. Lacki, Phys. Rev. D 88, 121301 (2013), arXiv:1306.3417 [astro-ph.HE]

[158] A. Capanema, A. Esmaili, and K. Murase, Phys. Rev. D 101, 103012 (2020), arXiv:2002.07192 [hep-ph]

[159] N. Gupta, Astropart. Phys. 48, 75 (2013), arXiv:1305.4123 [astro-ph.HE],

[160] J. C. Joshi, W. Winter, and N. Gupta, Mon. Not. Roy. Astron. Soc. 439, 3414 (2014), [Erratum: Mon.Not.Roy.Astron.Soc. 446, 892 (2014)], arXiv:1310.5123 [astro-ph.HE]

[161] M. Ahlers and K. Murase, Phys. Rev. D 90, 023010 (2014), arXiv:1309.4077 [astro-ph.HE]

[162] K. Mannheim, R. J. Protheroe, and J. P. Rachen, Phys. Rev. D 63, 023003 (2001), arXiv:astro-ph/9812398.

[163] V. S. Berezinsky and G. T. Zatsepin, Usp. Fiz. Nauk 122, 3 (1977)

[164] A. Neronov and D. Semikoz, Astron. Astrophys. 633, A94 (2020), arXiv:1907.06061 [astro-ph.HE]

[165] H. P. Dembinski, R. Engel, A. Fedynitch, T. Gaisser, F. Riehn, and T. Stanev, PoS ICRC2017, 533 (2018), arXiv:1711.11432 [astro-ph.HE]

[166] M. Kachelrieß, O. Kalashev, S. Ostapchenko, and D. V. Semikoz, Phys. Rev. D 96, 083006 (2017), arXiv:1704.06893 [astro-ph.HE]

[167] M. Ackermann et al. (Fermi-LAT), Phys. Rev. Lett. 116, 151105 (2016), arXiv:1511.00693 [astro-ph.CO]

[168] E. Roulet, JCAP 08, 009, arXiv:2105.12506 [astroph.HE]

[169] S. Hummer, M. Maltoni, W. Winter, and C. Yaguna, Astropart. Phys. 34, 205 (2010), arXiv:1007.0006 [astroph.HE]

[170] M. Bustamante and I. Tamborra, Phys. Rev. D 102, 123008 (2020), arXiv:2009.01306 [astro-ph.HE]

[171] K. Ryabtsev and S. Troitsky, In progress (2021).

[172] L. A. Anchordoqui, H. Goldberg, F. Halzen, and T. J. Weiler, Phys. Lett. B 593, 42 (2004), arXiv:astro$\mathrm{ph} / 0311002$

[173] M. Kachelriess and D. V. Semikoz, Phys. Lett. B 634, 143 (2006), arXiv:astro-ph/0510188

[174] V. Ptuskin, S. Rogovaya, and V. Zirakashvili, Adv. Space Res. 51, 315 (2013), arXiv:1105.4491 [astroph.CO]

[175] O. E. Kalashev, K. V. Ptitsyna, and S. V. Troitsky, Phys. Rev. D 86, 063005 (2012), arXiv:1207.2859 [astroph.HE]

[176] S. L. Dubovsky, P. G. Tinyakov, and I. I. Tkachev, Phys. 
Rev. Lett. 85, 1154 (2000), arXiv:astro-ph/0001317

[177] P. Lipari, Phys. Rev. D 78, 083011 (2008) arXiv:0808.0344 [astro-ph]

[178] A. Palladino, A. van Vliet, W. Winter, and A. Franckowiak, Mon. Not. Roy. Astron. Soc. 494, 4255 (2020), arXiv:1911.05756 [astro-ph.HE].

[179] M. Ahlers and F. Halzen, Phys. Rev. D 90, 043005 (2014), arXiv:1406.2160 [astro-ph.HE]

[180] K. Murase and E. Waxman, Phys. Rev. D 94, 103006 (2016), arXiv:1607.01601 [astro-ph.HE]

[181] S. Ando, M. R. Feyereisen, and M. Fornasa, Phys. Rev. D 95, 103003 (2017), arXiv:1701.02165 [astro-ph.HE]

[182] M. G. Aartsen et al. (IceCube), Eur. Phys. J. C 79, 234 (2019), arXiv:1811.07979 [hep-ph].

[183] A. Neronov and D. V. Semikoz, J. Exp. Theor. Phys. 131, 265 (2020) arXiv:1811.06356 [astro-ph.HE]

[184] C. Yuan, K. Murase, and P. Mészáros, Astrophys. J. 890, 25 (2020), arXiv:1904.06371 [astro-ph.HE].

[185] F. Capel, D. J. Mortlock, and C. Finley, Phys. Rev. D 101, 123017 (2020) arXiv:2005.02395 [astro-ph.HE]

[186] P. Mertsch, M. Rameez, and I. Tamborra, JCAP 03, 011, arXiv:1612.07311 [astro-ph.HE]

[187] M. G. Aartsen et al. (IceCube), JCAP 07, 042, arXiv:1911.11809 [astro-ph.HE]

[188] K. Fang, A. Banerjee, E. Charles, and Y. Omori, Astrophys. J. 894, 112 (2020), arXiv:2002.06234 [astroph.HE]

[189] V. Berezinsky, in Proceedings of the Neutrino-77 Conference, Moscow (1977) p. 177.

[190] D. Eichler, Astrophys. J. 232, 106 (1979).

[191] V. S. Berezinskii and V. L. Ginzburg, Mon. Not. Roy. Astron. Soc. 194, 3 (1981)

[192] K. Murase, Active Galactic Nuclei as High-Energy Neutrino Sources, in Neutrino Astronomy: Current Status, Future Prospects. Edited by Thomas Gaisser Albrecht Karle. Published by World Scientific Publishing Co. Pte. Ltd., 2017. ISBN \#9789814759410, pp. 15-31, edited by T. Gaisser and A. Karle (2017) pp. 15-31.

[193] P. Mészáros, Annual Review of Nuclear and Particle Science 67, 45 (2017), arXiv:1708.03577 [astro-ph.HE]

[194] M. Böttcher, Galaxies 7, 20 (2019), arXiv:1901.04178 [astro-ph.HE]

[195] M. Cerruti, in Proceedings of TAUP 2019 (2019) p. arXiv:1912.03666.

[196] M. C. Begelman, B. Rudak, and M. Sikora, Astrophys. J. 362, 38 (1990)

[197] F. W. Stecker, C. Done, M. H. Salamon, and P. Sommers, Phys. Rev. Lett. 66, 2697 (1991), [Erratum: Phys.Rev.Lett. 69, 2738 (1992)].

[198] K. Mannheim, T. Stanev, and P. L. Biermann, Astron. Astrophys. 260, L1 (1992).

[199] A. Y. Neronov and D. V. Semikoz, Phys. Rev. D 66, 123003 (2002), arXiv:hep-ph/0208248

[200] F. W. Stecker, Phys. Rev. D 88, 047301 (2013), arXiv:1305.7404 [astro-ph.HE].

[201] C. D. Dermer, K. Murase, and Y. Inoue, JHEAp 3-4, 29 (2014), arXiv:1406.2633 [astro-ph.HE],

[202] O. Kalashev, D. Semikoz, and I. Tkachev, J. Exp. Theor. Phys. 120, 541 (2015), arXiv:1410.8124 [astro-ph.HE]

[203] K. Ptitsyna and A. Neronov, Astron. Astrophys. 593, A8 (2016), arXiv:1510.04023 [astro-ph.HE]

[204] Y. Inoue, D. Khangulyan, S. Inoue, and A. Doi, arXiv e-prints , 1904.00554 (2019), arXiv:1904.00554 [astroph.HE]
[205] K. Murase, S. S. Kimura, and P. Meszaros, Phys. Rev. Lett. 125, 011101 (2020), arXiv:1904.04226 [astroph.HE]

[206] A. Kheirandish, K. Murase, and S. S. Kimura, Astrophys. J. 922, 45 (2021), arXiv:2102.04475 [astroph.HE]

[207] Y. Inoue, D. Khangulyan, and A. Doi, Galaxies 9, 36 (2021), arXiv:2105.08948 [astro-ph.HE]

[208] F. Halzen and E. Zas, Astrophys. J. 488, 669 (1997), arXiv:astro-ph/9702193

[209] A. Atoyan and C. D. Dermer, Phys. Rev. Lett. 87, 221102 (2001), arXiv:astro-ph/0108053

[210] A. Bykov, N. Gehrels, H. Krawczynski, M. Lemoine, G. Pelletier, and M. Pohl, Space Sci. Rev. 173, 309 (2012), arXiv:1205.2208 [astro-ph.HE]

[211] L. Sironi and A. Spitkovsky, Astrophys. J. 698, 1523 (2009), arXiv:0901.2578 [astro-ph.HE]

[212 M. Lemoine and E. Waxman, JCAP 11, 009 , arXiv:0907.1354 [astro-ph.HE].

[213] A. Neronov and D. Semikoz, JETP Lett. 113, 69 (2021), arXiv:2012.04425 [astro-ph.HE]

[214] A. Loeb and E. Waxman, JCAP 05, 003, arXiv:astro$\mathrm{ph} / 0601695$

[215] K. Bechtol, M. Ahlers, M. Di Mauro, M. Ajello, and J. Vandenbroucke, Astrophys. J. 836, 47 (2017), arXiv:1511.00688 [astro-ph.HE]

[216] M. D. Kistler, (2015), arXiv:1511.01530 [astro-ph.HE].

[217] K. Murase, S. Inoue, and S. Nagataki, Astrophys. J. Lett. 689, L105 (2008), arXiv:0805.0104 [astro-ph].

[218] K. Kotera, D. Allard, K. Murase, J. Aoi, Y. Dubois, T. Pierog, and S. Nagataki, Astrophys. J. 707, 370 (2009), arXiv:0907.2433 [astro-ph.HE]

[219] K. Fang and K. Murase, Nature Phys. 14, 396 (2018), arXiv:1704.00015 [astro-ph.HE]

[220] K. Murase, PoS ICRC2019, 965 (2020), arXiv:1912.05764 [astro-ph.HE]

[221] A. Ambrosone, M. Chianese, D. F. G. Fiorillo, A. Marinelli, G. Miele, and O. Pisanti, Mon. Not. Roy. Astron. Soc. 503, 4032 (2021), arXiv:2011.02483 [astroph.HE]

[222] P. Meszaros and E. Waxman, Phys. Rev. Lett. 87, 171102 (2001), arXiv:astro-ph/0103275

223] S. Razzaque, P. Meszaros, and E. Waxman, Phys. Rev. Lett. 93, 181101 (2004), [Erratum: Phys.Rev.Lett. 94, 109903 (2005)], arXiv:astro-ph/0407064.

[224] S. Ando and J. F. Beacom, Phys. Rev. Lett. 95, 061103 (2005), arXiv:astro-ph/0502521

[225] N. Gupta and B. Zhang, Astropart. Phys. 27, 386 (2007), arXiv:astro-ph/0606744

[226] K. Murase and K. Ioka, Phys. Rev. Lett. 111, 121102 (2013), arXiv:1306.2274 [astro-ph.HE]

[227] A. Bhattacharya, R. Enberg, M. H. Reno, and I. Sarcevic, JCAP 06, 034, arXiv:1407.2985 [astro-ph.HE].

[228] I. Tamborra and S. Ando, Phys. Rev. D 93, 053010 (2016), arXiv:1512.01559 [astro-ph.HE]

[229] N. Senno, K. Murase, and P. Meszaros, Phys. Rev. D 93, 083003 (2016), arXiv:1512.08513 [astro-ph.HE].

[230] D. Xiao, P. Mészáros, K. Murase, and Z.-g. Dai, Astrophys. J. 826, 133 (2016), arXiv:1604.08131 [astroph.HE]

[231] P. B. Denton and I. Tamborra, JCAP 04, 058, arXiv:1802.10098 [astro-ph.HE].

[232] H.-N. He, A. Kusenko, S. Nagataki, Y.-Z. Fan, and D.-M. Wei, Astrophys. J. 856, 119 (2018), 
arXiv:1803.07478 [astro-ph.HE]

[233] M. Ahlers and L. Halser, Mon. Not. Roy. Astron. Soc. 490, 4935 (2019) arXiv:1908.06953 [astro-ph.HE]

[234] X.-Y. Wang, R.-Y. Liu, Z.-G. Dai, and K. S. Cheng, Phys. Rev. D 84, 081301 (2011), arXiv:1106.2426 [astroph.HE].

[235] X.-Y. Wang and R.-Y. Liu, Phys. Rev. D 93, 083005 (2016), arXiv:1512.08596 [astro-ph.HE]

[236] L. Dai and K. Fang, Mon. Not. Roy. Astron. Soc. 469, 1354 (2017), arXiv:1612.00011 [astro-ph.HE]

[237] N. Senno, K. Murase, and P. Meszaros, Astrophys. J. 838, 3 (2017) arXiv:1612.00918 [astro-ph.HE]

[238] C. Lunardini and W. Winter, Phys. Rev. D 95, 123001 (2017), arXiv:1612.03160 [astro-ph.HE]

[239] C. Guépin, K. Kotera, E. Barausse, K. Fang, and K. Murase, Astron. Astrophys. 616, A179 (2018), [Erratum: Astron.Astrophys. 636, C3 (2020)], arXiv:1711.11274 [astro-ph.HE]

[240] D. Biehl, D. Boncioli, C. Lunardini, and W. Winter, Sci. Rep. 8, 10828 (2018), arXiv:1711.03555 [astro-ph.HE].

[241] K. Murase, S. S. Kimura, B. T. Zhang, F. Oikonomou and M. Petropoulou, Astrophys. J. 902, 108 (2020) arXiv:2005.08937 [astro-ph.HE].

[242] A. Gupta, S. Mathur, Y. Krongold, F. Nicastro, and M. Galeazzi, Astrophys. J. Lett. 756, L8 (2012), arXiv:1205.5037 [astro-ph.HE]

[243] N. Martynenko, (2021), arXiv:2105.02557 [astroph.GA]

[244] R. Feldmann, D. Hooper, and N. Y. Gnedin, Astrophys. J. 763, 21 (2013), arXiv:1205.0249 [astro-ph.HE]

[245] A. M. Taylor, S. Gabici, and F. Aharonian, Phys. Rev. D 89, 103003 (2014), arXiv:1403.3206 [astro-ph.HE]

[246] O. Kalashev and S. Troitsky, Phys. Rev. D 94, 063013 (2016), arXiv:1608.07421 [astro-ph.HE]

[247] S. Recchia, S. Gabici, F. A. Aharonian, and V. Niro, Astrophys. J. 914, 135 (2021), arXiv:2101.05016 [astroph.HE]

[248] G. Bertone and T. Tait, M. P., Nature 562, 51 (2018), arXiv:1810.01668 [astro-ph.CO]

[249] P. H. Frampton and S. L. Glashow, Phys. Rev. Lett. 44, $1481(1980)$

[250] J. R. Ellis, T. K. Gaisser, and G. Steigman, Nucl. Phys. B 177, 427 (1981)

[251] J. R. Ellis, G. B. Gelmini, J. L. Lopez, D. V. Nanopoulos, and S. Sarkar, Nucl. Phys. B 373, 399 (1992)

[252] V. S. Berezinsky, Nucl. Phys. B 380, 478 (1992)

[253] V. Berezinsky, M. Kachelriess, and A. Vilenkin, Phys.
Rev. Lett. 79, 4302 (1997), arXiv:astro-ph/9708217.

[254] V. A. Kuzmin and V. A. Rubakov, Phys. Atom. Nucl. 61, 1028 (1998), arXiv:astro-ph/9709187.

[255] B. Feldstein, A. Kusenko, S. Matsumoto, and T. T. Yanagida, Phys. Rev. D 88, 015004 (2013), arXiv:1303.7320 [hep-ph].

[256] A. Esmaili and P. D. Serpico, JCAP 11, 054, arXiv:1308.1105 [hep-ph]

[257] C. A. Argüelles, A. Kheirandish, and A. C. Vincent, Phys. Rev. Lett. 119, 201801 (2017), arXiv:1703.00451 [hep-ph]

[258] Y. Sui and P. S. Bhupal Dev, JCAP 07, 020 arXiv:1804.04919 [hep-ph]

[259] K. V. Berghaus, M. D. Diamond, and D. E. Kaplan, JHEP 05, 145 , arXiv:1811.04939 [hep-ph]

[260] M. Chianese, D. F. G. Fiorillo, G. Miele, S. Morisi, and O. Pisanti, JCAP 11, 046, arXiv:1907.11222 [hep-ph].

[261] M. Y. Kuznetsov, JETP Lett. 105, 561 (2017) arXiv:1611.08684 [astro-ph.HE]

[262] M. Kachelriess, O. E. Kalashev, and M. Y. Kuznetsov, Phys. Rev. D 98, 083016 (2018), arXiv:1805.04500 astro-ph.HE]

[263] M. Pandey, D. Majumdar, A. Halder, and S. Banerjee, Phys. Lett. B 797, 134910 (2019) arXiv:1905.08662 [hep-ph].

[264] O. Kalashev, M. Kuznetsov, and Y. Zhezher, JCAP 11, 016 arXiv:2005.04085 [astro-ph.HE]

[265] A. M. Bykov, D. C. Ellison, P. E. Gladilin, and S. M. Osipov, Mon. Not. Roy. Astron. Soc. 453, 113 (2015) arXiv:1507.04018 [astro-ph.HE],

[266] A. M. Bykov, Astron. Astrophys. Rev. 22, 77 (2014) arXiv:1511.04608 [astro-ph.HE]

[267] A. M. Bykov, D. C. Ellison, P. E. Gladilin, and S. M. Osipov, Adv. Space Res. 62, 2764 (2018), arXiv:1706.01135 [astro-ph.HE]

[268] A. M. Bykov, A. Marcowith, E. Amato, M. E. Kalyashova, J. M. D. Kruijssen, and E. Waxman, Space Sci. Rev. 216, 42 (2020), arXiv:2003.11534 [astroph.HE]

[269] M. Bouyahiaoui, M. Kachelrieß, and D. Semikoz, PoS ICRC2021, 999 (2021), arXiv:2105.13378 [astroph.HE]

[270] A. M. Bykov, A. E. Petrov, M. E. Kalyashova, and S. V. Troitsky, Astrophys. J. Lett. 921, L10 (2021), arXiv:2110.11189 [astro-ph.HE]

[271] A. Gallo Rosso, C. Mascaretti, A. Palladino, and F. Vissani, Eur. Phys. J. Plus 133, 267 (2018), arXiv:1806.06339 [astro-ph.HE] 